\title{
Multiscale thermomechanical contact: Computational homogenization with isogeometric analysis
}

\author{
İ. Temizer ${ }^{*} \dagger$ \\ Department of Mechanical Engineering, Bilkent University, 06800 Ankara, Turkey
}

\begin{abstract}
SUMMARY
A computational homogenization framework is developed in the context of the thermomechanical contact of two boundary layers with microscopically rough surfaces. The major goal is to accurately capture the temperature jump across the macroscopic interface in the finite deformation regime with finite deviations from the equilibrium temperature. Motivated by the limit of scale separation, a two-phase thermomechanically decoupled methodology is introduced, wherein a purely mechanical contact problem is followed by a purely thermal one. In order to correctly take into account finite size effects that are inherent to the problem, this algorithmically consistent two-phase framework is cast within a self-consistent iterative scheme that acts as a first-order corrector. For a comparison with alternative coupled homogenization frameworks as well as for numerical validation, a mortar-based thermomechanical contact algorithm is introduced. This algorithm is uniformly applicable to all orders of isogeometric discretizations through non-uniform rational B-spline basis functions. Overall, the two-phase approach combined with the mortar contact algorithm delivers a computational framework of optimal efficiency that can accurately represent the geometry of smooth surface textures. Copyright () 2013 John Wiley \& Sons, Ltd.
\end{abstract}

Received 2 October 2012; Revised 21 September 2013; Accepted 26 October 2013

KEY WORDS: contact resistance; finite deformations; thermomechanical contact; mortar method; isogeometric analysis; computational homogenization

\section{INTRODUCTION}

Almost all engineering surfaces are significantly rough on the microscale. Therefore, the modeling and simulation of an engineering design can critically rely on accurately capturing the effect of microscale roughness on the macroscale response. This effect is typically reflected through an appropriate modeling of contact interface boundary conditions. Because these boundary conditions drive the overall bulk response, it is important to develop appropriate scale transition techniques for a predictive macroscopic framework. Examples to multiscale contact interface phenomena of interest include classical tribological phenomena such as friction [1] and lubrication [2]. Another classical phenomenon is the so-called thermal contact resistance [3], which essentially models the observed temperature jump across the macroscopic contact interface. Technologically, this resistance plays an important role in the design of modern electronic devices. An outstanding example is the packaging problem for microprocessors where multiple contact interfaces exist. Because novel processors can easily dissipate power at more than $1 \mathrm{~W} / \mathrm{mm}^{2}$, any additional interface resistance due to roughness significantly contributes to overall heating, which, in turn, directly impacts the efficiency and the functionality of the device [4]. In order to reduce this undesirable macroscopic effect of rough contact interfaces, thermal interface materials (TIMs) are typically employed in electronics packaging [5]. TIMs could be gels, elastomeric pads and more recently nanowire forests [6]. In

*Correspondence to: İ. Temizer, Department of Mechanical Engineering, Bilkent University, 06800 Ankara, Turkey.

†E-mail: temizer@bilkent.edu.tr. 
all cases, they constitute a highly deformable medium that fills up the gaps between the surfaces, thereby leading to a continuous conduction domain and, hence, to a lower macroscopic resistance. One goal of this work is to develop a framework that is suitable for the modeling and analysis of interfaces with TIMs. From a broader perspective, a computational approach will be developed that is suitable for microscopically rough contact interfaces, which are subjected to finite deformations and finite deviations from the equilibrium temperature.

The following series of modeling choices will be made in order to develop a multiscale approach. First, because of the multiscale nature of the problem, the interaction between the surfaces may be modeled starting from the atomic scale $[7,8]$. In this work, emphasis will be on the transition from one continuum scale to another. Moreover, it is well known that the thermal interaction between surfaces in proximity, rough or not, is governed not only by contact but also by radiation as well as convection through an interstitial fluid medium [3,9], the latter usually being constrained to nonstandard conduction in significantly small gaps [10]. The majority of works addressing the class of problems that are of concern, on the other hand, concentrate on what appears to be the more dominant factor, namely the contact resistance. This will also be the emphasis of this work in order to establish a predictive framework for the problem at hand. Within these modeling choices, it is clear that the major challenge to the problem is the difference in the scales of the device to be analyzed and the roughness on the microscale. Homogenization appears as a natural methodology to link these two scales. Indeed, most approaches to the modeling of thermal contact resistance have been based on some form of analytical homogenization. The literature on this approach is extremely rich. Here, one can mention the reference works $[3,11]$ as well as some representative recent studies $[9,10,12-16]$ where detailed references may be found. Homogenization approaches that are based on analytical techniques have the major advantage that they can elegantly incorporate the effect of arbitrarily rough surfaces across multiple scales and can match experimental data quantitatively and qualitatively well. For this reason, they continue to constitute the majority of contributions that address thermal contact resistance modeling. However, they are unable to model the exact geometry of roughness at any one scale or the detailed asperity interactions, in particular when these interactions are nonlinear as in the finite deformation regime with finite deviations from the equilibrium temperature. Computational homogenization techniques are more appropriate for the latter purpose. A comparison of analytical and computational homogenization techniques pertaining to the present problem may be found in [17]. There are few computational homogenization approaches in the literature, see for instance [18-20]. Although randomly rough topographies have also been incorporated into these frameworks with accurate resolutions of the contact interface, they have been limited to small deformations. A finite deformation methodology has been proposed and investigated in the context of periodic [21] and random [17] contact microstructures, which will be taken as the basis of the present work. Therein, one of the surfaces was modeled as a smooth and rigid heat bath. The major extension pursued in this work is to drop this considerably simplifying assumption.

The construction of an appropriate computational thermomechanical contact homogenization framework will be realized in three major steps. In a first step, Section 2 introduces the homogenization of an entire boundary layer where absolute size effects are inherent to the problem. Subsequently, the approach is refined toward the homogenization of the contact interactions only, which will eventually recover some of the ideas introduced in earlier work. Finally, certain size effects that are implied by this framework but that are not entirely physical will be pointed out. Section 3 aims to resolve the issue of these non-physical size effects through a two-phase computational homogenization framework that is motivated by the scale separation limit. Highlighting physical size effects that are inherent to the multiscale problem at hand, this framework is then complemented by a self-consistent scheme that introduces a first-order corrector to account for the lack of asymptotic scale separation. The resulting approach calls for a numerically robust and efficient thermomechanical contact algorithm. The construction and discussion of such an algorithm, in light of recent developments, are the subject of Section 4 where the relevant literature review is provided. Here, the entire approach is cast within an isogeometric framework that has the significant advantage of being able to accurately represent smooth interface textures and contact geometries in addition to guaranteeing the uniform applicability of the contact algorithm to all orders of non-uniform rational 
B-spline (NURBS) discretizations. Section 5 addresses all the issues raised throughout the theoretical developments of the earlier sections and demonstrates the quantitative and qualitative accuracy of the homogenization approach as well as the numerical robustness and efficiency of the computational framework. An outlook toward the further development of the advocated approach for realistic thermal contact interfaces is outlined in the conclusion.

\section{HOMOGENIZATION WITH SIZE EFFECTS}

\subsection{Dissipation in a contact boundary layer}

The approach developed in an earlier work is first extended to the contact of two rough and deformable boundary layers. The aim is to highlight size effects which are intrinsic to this homogenization methodology. The continuum framework for the problem is briefly reviewed in Appendix A.

The macroscopic homogenized response will be extracted from the microscale thermomechanical contact analysis of two samples with reference (spatial) configurations $\mathcal{C}_{o}^{(I)}\left(\mathcal{C}^{(I)}\right)$ from the boundary layers $\mathcal{B}^{(I)}$ of two contacting bodies (Figure 1). The starting point is the monitoring of the thermal dissipation per unit volume of the spatial configuration:

$$
\mathcal{D}=-\boldsymbol{q} \cdot \boldsymbol{g} / \theta=-\boldsymbol{q} \cdot \operatorname{grad}[\ln \theta] .
$$

Here, $\boldsymbol{q}$ is the heat flux, which defines the normal heat flux $h=-\boldsymbol{q} \cdot \boldsymbol{n}$ on a surface with outward unit normal $\boldsymbol{n}, \theta$ is the temperature and $\boldsymbol{g}=\operatorname{grad}[\theta]$ is the temperature gradient. The total volumetric dissipation $\Phi_{v}$ may be expressed in terms of boundary data as follows:

$$
\begin{aligned}
\Phi_{v} & =\sum_{I} \int_{\mathcal{C}^{(I)}} \mathcal{D} \mathrm{d} v=\sum_{I} \int_{\partial \mathcal{C}^{(I)}} h \ln \theta \mathrm{d} a \\
& =\sum_{I}\left(\int_{\partial \mathcal{C}^{(I), e}} h \ln \theta \mathrm{d} a+\int_{\partial \mathcal{C}^{(I), r}} h \ln \theta \mathrm{d} a+\int_{\partial \mathcal{C}^{(I), i}} h \ln \theta \mathrm{d} a\right) .
\end{aligned}
$$

In order to isolate roughness effects in a first step, it is assumed that contacting points on the microscale pick the same temperature-the case with microscopic contact resistance will be addressed later. It is well-known that there is a possible non-existence of solutions for thermomechanical contact whenever such a temperature continuity assumption is made [22]. Nevertheless, this problem will not be encountered in the class of problems considered below. The integrals over the rough surfaces now sum to zero because either $h$ vanishes where there is no contact on $\partial \mathcal{C}^{(I), r}$ or it is of equal magnitude but with opposite sign on the contacting portions. On the other hand, each integral over the internal surfaces $\partial \mathcal{C}^{(I), i}$ also vanishes owing to the boundary conditions $(\mathrm{BCs})$ employed. Here, only the thermal BCs are stated. By decomposing $\partial \mathcal{C}^{(I), i}$ to opposing surfaces $\partial \mathcal{C}^{(I), i+}$ and $\partial \mathcal{C}^{(I), i-}, \mathrm{BCs}$ are either of the linear (LN) or periodic (PR) type:

Linear: $h=0$, Periodic: $\theta^{+}=\theta^{-}$and $h^{+}=-h^{-}$.

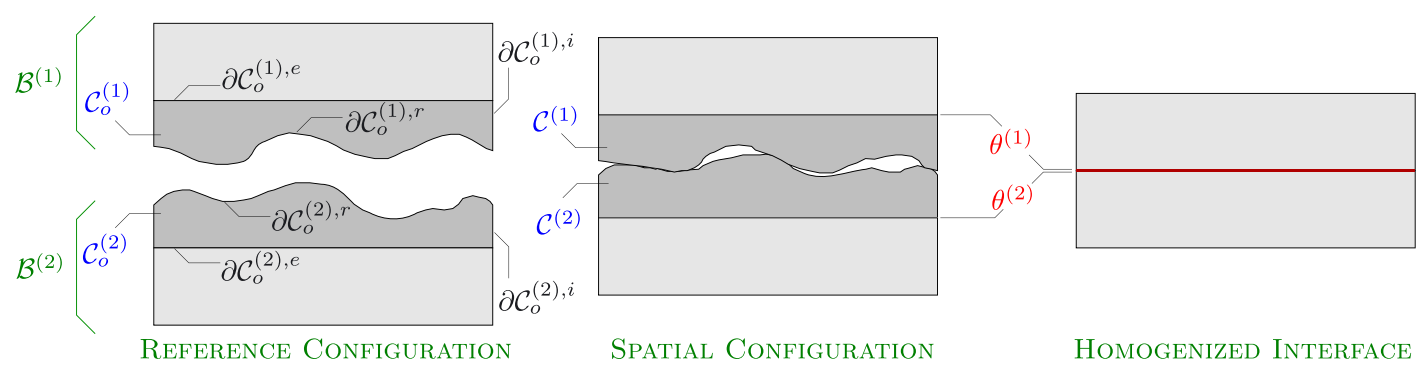

Figure 1. The setup for the homogenization of an entire boundary layer is depicted, within which one identifies samples $\mathcal{C}_{o}^{(I)}$ from the boundary layers $\mathcal{B}^{(I)}$ in the vicinity of the contact interface. 
Finally, the flux and temperature distributions on the observable test surfaces $\partial \mathcal{C}^{(I), e}$ are uniform, and the surfaces remain flat under the imposed macroscopic pressure for samples of sufficient height. The respective distributions are denoted by $\theta^{(I)}$ and $h^{(I)}$. The flux integrals must match the macroscopic normal heat flux $\bar{h}$ via

$$
-\int_{\partial \mathcal{C}^{(1), e}} h \mathrm{~d} a=\int_{\partial \mathcal{C}^{(2), e}} h \mathrm{~d} a=\bar{h}|\mathcal{A}| .
$$

Here, $|\mathcal{A}|$ is the area of $\partial \mathcal{C}^{(I), e}$ and represents the macroscopic contact area associated with these samples. Consequently, the dissipation per unit macroscopic contact area reads

$$
\phi_{v}=\Phi_{v} /|\mathcal{A}|=-\bar{h}\left(\ln \theta^{(1)}-\ln \theta^{(2)}\right) .
$$

\subsection{Macroscopic temperature jump}

2.2.1. Homogenization of the entire boundary layer. There are two cases of interest associated with the present setup. In the first case, the entire contact boundary layer with a finite thickness is homogenized toward a macroscopic contact interaction (Figure 1). Within a standard computational contact mechanics setting on the macroscale, the temperature $\bar{\theta}$ of a point on the slave surface and the contact surface temperature $\bar{\theta}_{c}$ at its projection onto the master are known. The macroscopic temperature jump is of finite magnitude

$$
\bar{\vartheta}_{c}=\bar{\theta}-\bar{\theta}_{c}
$$

and delivers the normal heat flux $\bar{h}$, positive values denoting flow from master to slave, via

$$
\bar{h}=-\bar{k}_{c} \bar{\vartheta}_{c}
$$

where $\bar{k}_{c}$ is the macroscopic contact conductance or $\bar{r}_{c}=\bar{k}_{c}^{-1}$ is the resistance. The unknown conductance is to be determined by homogenization. Presently, this is straightforward in view of the procedure leading to (2.5). Assuming $\mathcal{B}^{(2)}$ is the master, one prescribes $\theta^{(2)}$ to $\bar{\theta}_{c}$ and $\theta^{(1)}$ to $\bar{\theta}$ and measures the corresponding flux $\bar{h}$ via (2.4). In other words,

$$
\bar{\vartheta}_{c}=\theta^{(1)}-\theta^{(2)} \text {. }
$$

Alternatively, if one wishes to monitor the trends in the macroscopic response at constant $\bar{h}$, then $\theta^{(1)}$ may be measured for prescribed $\bar{h}$. In either scenario, $\bar{k}_{c}$ is easily determined, and the associated macroscopic contact dissipation reads

$$
\bar{\phi}_{c}=-\bar{h}\left(\ln \bar{\theta}-\ln \bar{\theta}_{c}\right),
$$

which, by construction, matches the microscale one (2.5). Therefore, an alternative starting point would be to enforce this equality, which then induces the homogenization procedure described. In other words, homogenization should preserve dissipation through the scale transition. Clearly, the thicker the boundary layer, the higher the dissipation so that this case intrinsically has size dependence: $\bar{\vartheta}_{c}$ increases with increasing thickness. Moreover, $\bar{k}_{c}$ is expected to be highly dependent on the deformation of the boundary layer and the magnitude of the heat flux, due to thermomechanical coupling and the evolution of the contact interface.

2.2.2. Homogenization of the contact interactions. In the second case, only the contact interactions associated with the previous setup are homogenized. It is assumed that the macroscopic normal heat flux $\bar{h}$ is imposed. The idea is to reduce the original problem to one where the surfaces are macroscopically smooth, yet they display a contact resistance. To characterize this additional resistance, the original heterogeneous and homogenized problems must be compared with a homogeneous problem where the surfaces are microscopically flat, and therefore, no macroscopic resistance is displayed. Because the homogeneous and homogenized problems have flat contact surfaces, it is sufficient to represent them via two contacting bars (Figure 2). 


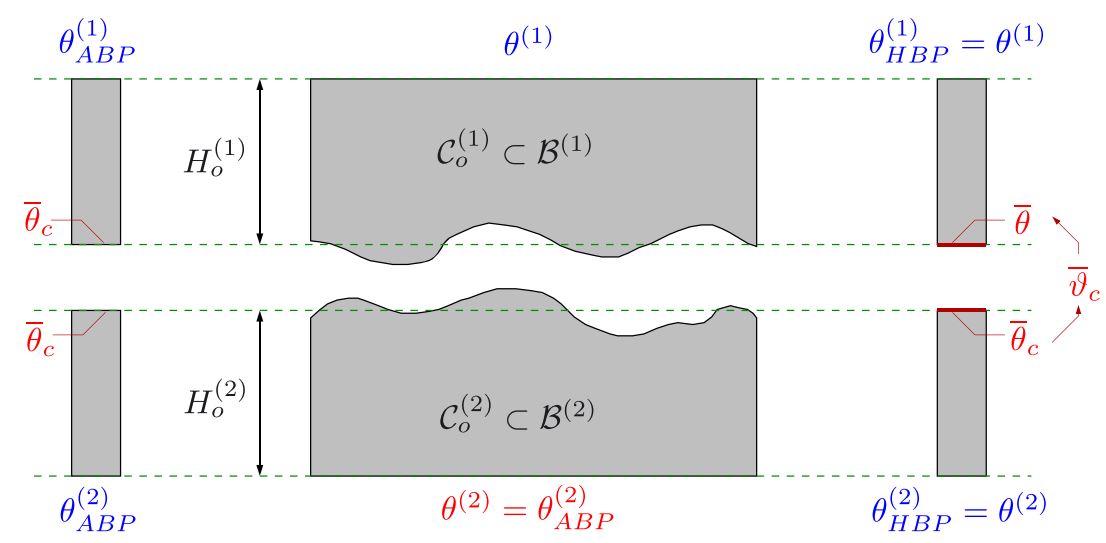

Figure 2. The three problems designed for the homogenization of the contact interactions are depicted. Here, it is assumed that $\mathcal{B}^{(2)}$ is associated with the master surface on the macroscale. The initial heights $H_{O}^{(I)}$ of the samples in the three problems should match by construction. For the samples $\mathcal{C}_{o}^{(I)}$, the heights are measured with respect to the mean planes of the rough surfaces. See also Figure 1 for boundary designations.

The dissipation in the homogeneous accompanying bar problem (ABP) may be decomposed as

$$
\begin{aligned}
\phi_{v}^{\mathrm{ABP}} & =-\bar{h}\left(\ln \theta_{\mathrm{ABP}}^{(1)}-\ln \theta_{\mathrm{ABP}}^{(2)}\right) \\
& =-\bar{h}\left(\ln \theta_{\mathrm{ABP}}^{(1)}-\ln \bar{\theta}_{c}\right)-\bar{h}\left(\ln \bar{\theta}_{c}-\ln \theta_{\mathrm{ABP}}^{(2)}\right) .
\end{aligned}
$$

This decomposition then suggests that the unknown $\theta_{\mathrm{ABP}}^{(I)}$ are computed by imposing the master temperature $\bar{\theta}_{c}$ on the contacting surfaces together with $\bar{h}$ on the external ones.

The dissipation in the homogenized bar problem (HBP) may be decomposed as

$$
\begin{aligned}
\phi_{v}^{\mathrm{HBP}} & =-\bar{h}\left(\ln \theta_{\mathrm{HBP}}^{(1)}-\ln \theta_{\mathrm{HBP}}^{(2)}\right) \\
& =-\bar{h}\left(\ln \theta_{\mathrm{HBP}}^{(1)}-\ln \bar{\theta}\right)+\bar{\phi}_{c}-\bar{h}\left(\ln \bar{\theta}_{c}-\ln \theta_{\mathrm{HBP}}^{(2)}\right),
\end{aligned}
$$

where $\bar{\phi}_{c}$ is now associated with the contact dissipation due to roughness alone. Here, $\mathcal{B}^{(2)}$ was assumed to be the master. This problem is never solved, unless for validation, although $\theta_{\mathrm{HBP}}^{(I)}$ need to be related to the original heterogeneous problem (OHP) for which the dissipation is of the form (2.5). Because $\bar{h}$ is prescribed, either $\theta^{(1)}$ or $\theta^{(2)}$ can be set in OHP, and the other must be computed. Simultaneously, $\bar{\theta}_{c}$ must be projected into the OHP. One choice would be to assign the macroscopic contact temperature $\bar{\theta}_{c}$ to the surface that is associated with the master. Presently, this corresponds to setting $\theta=\bar{\theta}_{c}$ on $\partial \mathcal{C}^{(2), r}$. However, this is unrealistic because the temperature fields on the rough surfaces are highly oscillatory. The choice made here mimics the case where the boundary layer $\mathcal{B}^{(2)}$ is rigid with a smooth surface. In that case, $\theta=\bar{\theta}_{c}$ on $\partial \mathcal{C}^{(2), r}$ essentially implies $\theta=\theta_{\mathrm{ABP}}^{(2)}$ on $\partial \mathcal{C}^{(2), e}$. Enforcing this equality even for the non-rigid $\mathcal{B}^{(2)}$ emulates this situation and offers an indirect method of projecting the macroscopic contact temperature onto the contact interface:

$$
\bar{\theta}_{c} \text {-Projection : if } \mathcal{B}^{(J)} \text { is the master, then set } \theta=\theta_{\mathrm{ABP}}^{(J)} \text { on } \partial \mathcal{C}^{(J), e} \text {. }
$$

The complementary condition on the slave is to enforce $\bar{h}$, for example, $h=-\bar{h}$ on $\partial \mathcal{C}^{(1), e}-$ see (2.4).

Once $\bar{\theta}_{c}$ is projected as described and $\theta^{(I)}$ in (2.5) are determined, the remaining step is to identify the intrinsic unknown $\bar{\vartheta}_{c}$ in $\bar{\phi}_{c}$ of (2.11). Proposing that the additional dissipation $\bar{\phi}_{c}$ in HBP, which has the explicit form (2.9), is due to the difference in the dissipations of OHP and ABP, that is,

$$
\bar{\phi}_{c}=\phi_{v}-\phi_{v}^{\mathrm{ABP}}
$$


one obtains the explicit expression

$$
\bar{\vartheta}_{c}^{(I)}=\left(\theta^{(I)} / \theta_{\mathrm{ABP}}^{(I)}-1\right) \bar{\theta}_{c},
$$

where the superscript denotes the macroscopic algorithmic designation of slave. This expression is entirely equivalent to the result obtained for a rigid smooth $\mathcal{B}^{(2)}$ at a constant temperature $\bar{\theta}_{c}$ [21]. Note that there is a sign difference: if $\bar{\vartheta}_{c}^{(1)}>0$, then $\bar{\vartheta}_{c}^{(2)}<0$. In order to obtain the result (2.14) according to this convention for the case when $\mathcal{B}^{(2)}$ is the slave $(I=2), \bar{\theta}$ and $\bar{\theta}_{c}$ must be switched in (2.9) and in (2.11) for completeness, for use in (2.13).

Incorporating microscopic contact resistance due to roughness on lower scales is straightforward. The resistance is simply omitted from ABP and incorporated only into OHP. The quantities $\bar{\vartheta}_{c}{ }^{(I)}$ then reflect the accumulated resistance due to roughness on multiple length scales [17].

2.2.3. Size effects. The choice of the master/slave on the macroscale should be properly reflected onto the microscale. For instance, when $\mathcal{B}^{(2)}$ is the master, then prescribing $\theta=\theta_{\mathrm{ABP}}^{(1)}$ on $\partial \mathcal{C}^{(1), e}$ is an incorrect procedure. This would lead to significantly different temperature distributions in both samples, and because of thermomechanical coupling, $\left|\bar{\vartheta}_{c}^{(1)}\right|=\left|\bar{\vartheta}_{c}^{(2)}\right|$ is not expected. However, this equality is not expected even without thermomechanical coupling. To see this, the physical setting is further simplified by assuming the following: (i) the thermal conductivities $k_{o}$ are temperature independent and equal, (ii) the samples are rigid with equal heights $H_{o}$, (iii) the surfaces are smooth but display a contact resistance such that at the prescribed $\bar{h}>0$, the microscopic temperature jump from $\mathcal{B}^{(2)}$ to $\mathcal{B}^{(1)}$ is $\vartheta_{c}>0$. In this simplified case, one does not expect an explicit effect of $\bar{\theta}_{c}$, and hence, its mere presence in (2.14) is questionable. In fact, if $\bar{g}=\bar{h} / k_{o}$ is the normal component of the temperature gradient within both boundary layers in the vicinity of the contact interface and $\mathcal{B}^{(2)}$ is the master with a surface temperature $\bar{\theta}_{c}$, then $\theta_{\mathrm{ABP}}^{(2)}=\theta^{(2)}=\bar{\theta}_{c}-\bar{g}_{o}, \theta_{\mathrm{ABP}}^{(1)}=\bar{\theta}_{c}+\bar{g} H_{o}$ and $\theta^{(1)}=\bar{\theta}_{c}+\vartheta_{c}+\bar{g} H_{o}$ such that $\bar{\theta}_{c}^{(1)}$ is obtained directly from (2.14). Similarly, if $\mathcal{B}^{(1)}$ is the master with the elevated surface temperature $\bar{\theta}_{c}^{\prime}=\bar{\theta}_{c}+\vartheta_{c}$, then $\theta_{\mathrm{ABP}}^{(1)}=\theta^{(1)}=\bar{\theta}_{c}^{\prime}+\bar{g} H_{o}$, $\theta_{\mathrm{ABP}}^{(2)}=\bar{\theta}_{c}^{\prime}-\bar{g} H_{o}$ and $\theta^{(2)}=\bar{\theta}_{c}-\bar{g} H_{o}$, delivering the expression for $\bar{\vartheta}_{c}^{(2)}$. The combined result is

$$
\left(1-\frac{\bar{g} H_{o}}{\bar{\theta}_{c}+\bar{g} H_{o}}\right) \vartheta_{c}=\bar{\vartheta}_{c}^{(1)} \leqslant \vartheta_{c} \leqslant-\bar{\vartheta}_{c}^{(2)}=\left(1+\frac{\bar{g} H_{o}}{{\overline{\theta^{\prime}}}_{c}-\bar{g} H_{o}}\right) \vartheta_{c} .
$$

Therefore, only in the limit as $H_{o} \rightarrow 0$ does (2.14) deliver the expected simple result $\left|\bar{\vartheta}_{c}^{(I)}\right|=\vartheta_{c}$, indicating a size effect, despite the fact that the macroscopic surface temperatures were kept the same (at $\bar{\theta}_{c}+\vartheta_{c}$ and $\bar{\theta}_{c}$ for $\mathcal{B}^{(1)}$ and $\mathcal{B}^{(2)}$, respectively) and only the macroscopic master/slave designation was switched. It is proposed that this effect and similar ones, which will be further demonstrated in the numerical investigations, are spurious in the sense that they do not reflect the macroscopic homogenized response to desired accuracy. An approach to alleviate this shortcoming, which is not as easy to detect when one surface is a rigid heat bath, is investigated next.

\section{A TWO-PHASE SELF-CONSISTENT FRAMEWORK}

\subsection{Limit of scale separation: algorithmic consistency}

Physically relevant samples have prescribed in-plane and out-of-plane statistical characteristics, such as the root-mean-square (RMS) roughness and autocorrelation. The limit of scale separation for the present problem is enforced by scaling down samples with given characteristics by a factor of $\varepsilon \rightarrow 0$. If the applied flux is simultaneously scaled by $\varepsilon^{-1}$, then the jump will remain at a constant value, essentially preserving all size effects. On the other hand, when the flux is a constant, the measured jump will vanish because $\varepsilon$ drives the surfaces to microscopic smoothness. To obtain a meaningful limit in this case, scaled temperature jumps

$$
\bar{\vartheta}_{c, \varepsilon}^{(I)}=\varepsilon^{-1} \bar{\vartheta}_{c}^{(I)}
$$


are monitored. These are expected to converge to a scale separation limit

$$
\lim _{\varepsilon \rightarrow 0}\left|\bar{\vartheta}_{c, \varepsilon}^{(I)}\right|=\bar{\vartheta}_{c, o}
$$

that is independent of which macroscopic surface is at $\bar{\theta}_{c}$. In order to build a basis for the verification of this expectation, it is necessary to go back to (2.1). For small $\varepsilon$, the deviations of the temperature from the contact temperature $\bar{\theta}_{c}$ are small. In this regime, the volumetric dissipation can be evaluated as in linear thermoelasticity where $\bar{\theta}_{c}$ serves as the reference temperature:

$$
\mathcal{D}=-\boldsymbol{q} \cdot \boldsymbol{g} / \bar{\theta}_{c} .
$$

This simplification leads to the replacement of $\ln \theta$ with $\theta / \bar{\theta}_{c}$ in all subsequent steps, thereby leading to the final result

$$
\bar{\vartheta}_{c}^{(I)}=\theta^{(I)}-\theta_{\mathrm{ABP}}^{(I)}
$$

As expected, this result can alternatively be directly obtained from the linearization of (2.14). Hence, it will be referred to as the linearized identification for the macroscopic temperature jump. This expression successfully delivers the expected response in the simplified test of Section 2.2.3 and agrees with some of the classical identifications of the macroscopic temperature jump [3].

In order to apply (3.4) within a computational setting, it is proposed that all temperaturedependent material properties be explicitly evaluated at the contact interface temperature $\bar{\theta}_{c}$ within the microscale thermomechanical contact analysis for measuring $\theta^{(I)}$ and $\theta_{\mathrm{ABP}}^{(I)}$. This modification reflects the macroscopic observation that as $\varepsilon \rightarrow 0$, the temperature within the material in the vicinity of the contact interface remains approximately equal to $\bar{\theta}_{c}$. This explicit enforcement of $\bar{\theta}_{c}$, similar to the ideas in $[23,24]$, decouples the micromechanical analysis into two phases:

(i) Mechanical phase: The macroscopic contact pressure is applied to the two samples within a purely mechanical contact problem to solve for the deformed configuration and thereby resolve the real contact area. Within this setup, all temperature-dependent material properties are evaluated at $\bar{\theta}_{c}$.

(ii) Thermal phase: On the frozen mechanical configuration with a resolved contact interface, a purely thermal problem is solved where the macroscopic normal heat flux $\bar{h}$ is enforced and the temperature jump $\bar{\vartheta}_{c}^{(I)}$ is measured via (3.4).

Within the mechanical phase, it is also possible to apply the macroscopic surficial stretch as pursued in earlier works. Additionally, note that a thermal contact formulation is still relevant because of the necessity of coupling the possibly non-matching discretizations on the two sides of the interface within the thermal phase. Finally, this two-phase framework delivers $\bar{\vartheta}_{c}^{(1)}=-\bar{\vartheta}_{c}^{(2)}$, the equality being a consequence of the explicit enforcement of $\bar{\theta}_{c}$. In other words, the overall framework is algorithmically consistent in the sense that the purely algorithmic choice of master and slave on the macroscale does not affect the outcome of the homogenization process.

\subsection{An application to textured interfaces}

As a special case of the scale separation limit, consider two textured surfaces that form a perfectly conforming resistive contact interface (Figure 3). Because contact resistance is already present, the macroscopic temperature jump converges to a non-zero limit even as $\varepsilon \rightarrow 0$. Presently, modeling and analysis will be carried out by mapping all quantities to the reference configuration of the interface-see also Section 4.1. Modeling the interface heat flux on the reference configuration by $h_{o}=-k_{c} \vartheta_{c}$, it is assumed that $\bar{h}_{o}<0$ and $\bar{\vartheta}_{c}=\bar{\vartheta}_{c}^{(1)}>0$. The microscopic conductance is modeled by the relatively general expression $k_{c}=k_{\theta} p_{N}^{\alpha}$, where $k_{\theta}$ and $\alpha$ are interface constants and $p_{N}$ is the contact pressure on the reference configuration. At scale separation, the temperature jump is uniform throughout the interface and therefore must correspond to $\bar{\vartheta}_{c}$. Making use of this observation together with (2.4) and a thermal balance on the sample, one obtains

$$
\bar{\vartheta}_{c}=-\frac{\bar{h}}{k_{\theta}\left[p_{N}^{\alpha}\right]},
$$




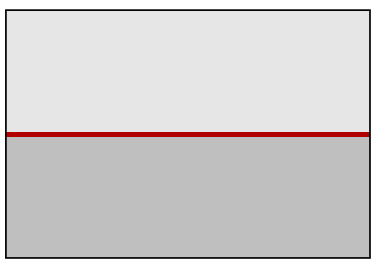

smooth resistive interface

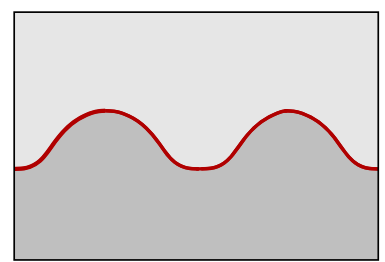

textured resistive interface

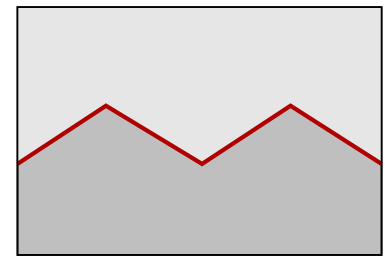

simple texture

Figure 3. Texturing of a perfectly conforming resistive interface.

where $\left[p_{N}^{\alpha}\right]=|\mathcal{A}|^{-1} \int_{\partial \mathcal{C}_{o}^{(1), r}} p_{N}^{\alpha} \mathrm{d} A$ has been introduced. Therefore, the calculation of $\bar{\vartheta}_{c}$ is based on a purely mechanical problem that delivers the pressure distribution at the interface. On the other hand, if the interface is smooth, then the setup simplifies to the test case of Section 2.2.3, delivering the jump for a smooth interface:

$$
\vartheta_{c}=-\frac{\bar{h}}{k_{\theta} \bar{p}_{N}^{\alpha}} .
$$

Combining these two expressions, one obtains

$$
\bar{\vartheta}_{c}=\frac{\bar{p}_{N}^{\alpha}}{\left[p_{N}^{\alpha}\right]} \vartheta_{c} .
$$

Clearly, because $\left[p_{N}^{\alpha}\right]$ is not necessarily equal to $\bar{p}_{N}^{\alpha}, \bar{\vartheta}_{c} \neq \vartheta_{c}$ in general. In fact, $\left[p_{N}^{\alpha}\right]>\bar{p}_{N}^{\alpha}$ is possible. In other words, texturing of perfectly conforming resistive interfaces may lead to a reduction in the observed macroscopic resistance. This observation will be verified numerically for three-dimensional random and periodic interface textures. The simple texture in Figure 3 demonstrates the idea. The pressure on the inclined planes is equal to the macroscopic pressure, which also corresponds to the pressure on the smooth interface. However, because the interface pressure is distributed on a larger area for the textured case, $\left[p_{N}^{\alpha}\right]=\left[\bar{p}_{N}^{\alpha}\right]>\bar{p}_{N}^{\alpha}$ is guaranteed, and the effect follows.

\subsection{Accounting for finite temperature changes}

The described micromechanical analysis of Section 3.1 has the significant advantage that it delivers a single, well-defined macroscopic temperature jump magnitude for a given microstructure and macroscopic control parameters. In particular, the result does not depend on arbitrary modeling parameters, such as the height of the samples beyond a certain value. Consequently, as the height of the sample is varied, the extracted macroscopic response rapidly converges to a limit. This is a significant advantage that avoids any ambiguity in the identification of the macroscopic interface resistance and is not strictly satisfied by (2.14). Numerical investigations will demonstrate the degree to which the two formulations deviate from each other.

There is, however, a shortcoming in the procedure described. As a special case, it is assumed that there is no microscale contact resistance. From a theoretical point of view, the described procedure is exact only when $\left|\bar{\vartheta}_{c}^{(I)}\right| / \bar{\theta}_{c} \ll 1$. However, the temperature jumps are negligibly small in this scenario. In other words, there would be no loss of accuracy in the macroscale thermomechanical contact analysis if the roughness-induced jump is omitted altogether. From a practical point of view, on the other hand, finite macroscopic temperature jumps are typically observed. This is in general true when the presence of microscale contact resistance is admitted. In this scenario, the assumption of both boundary layers being approximately at the constant temperature $\bar{\theta}_{c}$ is unrealistic. Instead, this condition holds only for the master. The slave boundary layer is approximately at $\bar{\theta}=\bar{\theta}_{c}+\bar{\vartheta}_{c}^{(I)}$. This condition is now introduced into an iterative two-phase micromechanical analysis as a first-order corrector through a self-consistent procedure: 
(i) Initial assumption: Evaluate all material properties at $\bar{\theta}_{c}$.

(ii) Two-phase analysis: Solve the mechanical phase followed by the thermal one to find $\bar{\vartheta}_{c}^{(I)}$.

(iii) First-order correction: Reevaluate the slave material properties at $\bar{\theta}=\bar{\theta}_{c}+\bar{\vartheta}_{c}^{(I)}$.

(iv) Self-consistency iterations: Check if $\bar{\vartheta}_{c}^{(I)}$ has converged. Otherwise, resolve.

The framework is depicted in Figure 4. In this work, the mechanical and thermal phases, although decoupled, are solved simultaneously by eliminating all coupling due to temperature-dependent material properties. Hence, a coupled thermomechanical contact algorithm was employed, which will be delineated in the following section.

Within this framework, $\bar{\vartheta}_{c}^{(1)} \neq-\bar{\vartheta}_{c}^{(2)}$ in general because the material properties of, say, $\mathcal{C}^{(1)}$ are evaluated at $\bar{\theta}=\bar{\theta}_{c}+\bar{\vartheta}_{c}$ in one case and at $\bar{\theta}_{c}$ in the other, thereby leading to different temperature distributions within the thermal phase from which $\bar{\vartheta}_{c}^{(I)}$ are measured. But this effect is not spurious, it is physical. Indeed, to obtain the same value with $\bar{\vartheta}_{c}^{(1)}$, the computation of $-\bar{\vartheta}_{c}^{(2)}$ requires setting the macroscopic contact surface temperature to $\bar{\theta}$. In this sense, the macroscopic algorithmic choice of master/slave does not influence the outcome of the homogenization process.

This updated two-phase framework with self-consistency retains the advantage of a uniquely identifiable macroscopic temperature jump while additionally accounting for finite temperature changes due to the observed jump. It should be noted that self-consistency is a minor requirement when the thermal conductivities of the involved materials are temperature independent. This is due to the small microstructural changes induced by the temperature-dependent mechanical properties of most materials. On the other hand, self-consistency can favorably improve the predictive capability of the framework in case of temperature-dependent thermal properties as to be demonstrated in the numerical investigations.

\section{MORTAR-BASED ISOGEOMETRIC THERMOMECHANICAL CONTACT}

In this section, a mortar-based isogeometric thermomechanical contact framework will be summarized. Numerical investigations will demonstrate the necessity of a robust contact formulation for the following: (i) the accurate resolution of the interface temperature field and (ii) the numerically efficient identification of the macroscopic response. The mortar-based approach to be developed to address this need is uniformly applicable to isogeometric discretizations of the test samples and the macroscopic bodies for all orders of NURBS basis functions; that is, no modifications are needed for higher-order basis functions that deliver an efficient resolution of smooth microscale and macroscale features. The distinguishing feature of NURBS basis functions that enables this uniform applicability is that they are non-negative so that their integrals over element

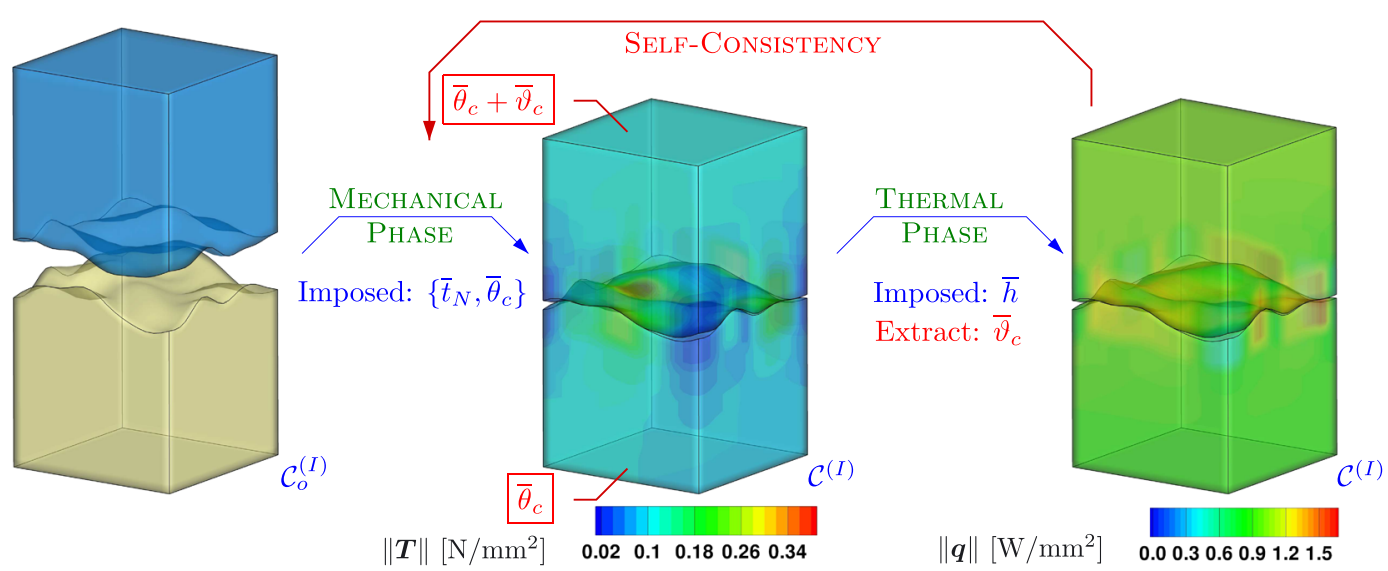

Figure 4. The two-phase computational contact homogenization framework is demonstrated. The selfconsistency iterations act as a first-order corrector that takes into account finite temperature changes. Here, $\boldsymbol{T}$ is the Cauchy stress and $\boldsymbol{q}$ is the heat flux. 
domains are guaranteed to be positive [25]. This feature, which is valid only for first-order Lagrange basis functions, hence requiring special care with quadratic Lagrange discretizations [26, 27], is intrinsically being made use of while summarizing the mortar-based framework. For various demonstrations of isogeometric contact and domain decomposition algorithms, see [28-33]. An important feature of these algorithms is that they are mostly based on the mortar method, which was shown to demonstrate various advantages over more classical contact algorithms even in the purely mechanical case, in particular for non-matching discretizations. For detailed discussions, the interested reader is referred to the extensive lists of references in these works-see also a recent review by [34]. Presently, the contributions by $[35,36]$ are specifically highlighted because they pertain to finite deformations, with and without friction, and employ an intermediate surface for integration as a main feature of mortar methods. On the other hand, mortar methods have been applied to thermomechanical contact and domain decomposition in few works [37-39]. In the following, an isogeometric thermomechanical contact treatment will be developed, based on earlier works on mortar methods. Although this approach does not make use of an intermediate integration surface, it will be shown to satisfy the requirements stated at the beginning of this section. The presentation will concentrate on thermal contact because its mechanical counterpart has been extensively investigated in the cited works.

\subsection{Thermal contact interface modeling}

In computational mechanics of frictionless thermomechanical contact, the standard contact contribution to the weak form of the energy balance is

$$
\delta \mathcal{G}_{\theta}^{c}=\int_{\Gamma_{o}^{c}} \delta \vartheta_{c} h_{o} \mathrm{~d} A,
$$

where $\Gamma_{o}^{c}$ is the contact interface $\Gamma^{c}$ mapped onto the referential surface of the slave. Here, $\delta \vartheta_{c}=\delta \theta-\delta \theta_{c}$ has been introduced for mere notational convenience, with $\theta$ as the temperature at a slave point and $\theta_{c}$ is the temperature at its projection onto the master. This notation, and the lack of an explicit notation for projected quantities, is used for compactness at the risk of oversimplification. In particular, the linearization $\Delta \vartheta_{c}$ is not equal to $\Delta \theta-\Delta \theta_{c}$ because of the missing derivatives associated with varying projection points. Computational and theoretical aspects of thermomechanical contact are discussed in the monographs by [40,41]. For early developments as well as theoretical and computational aspects, see also [42-44]. In particular, it is worth pointing out that a thermomechanical contact framework that provides more theoretical generality than the one employed here, which is captured as a special case, was presented in [43].

In order to model $h_{o}$, a thermal interface dissipation potential $\mathcal{H}_{o}\left(\vartheta_{c}, p_{N}\right)$ is introduced such that it delivers the contact heat flux:

$$
h_{o}\left(\vartheta_{c}, p_{N}\right)=\frac{\partial \mathcal{H}_{o}}{\partial \vartheta_{c}} .
$$

Two particular models for $\mathcal{H}_{o}$ are of interest:

(i) Constraint model: $\mathcal{H}_{o}=-\epsilon_{\theta} \vartheta_{c}^{2} / 2$.

Here, $\epsilon_{\theta}$ has the role of a penalty parameter that enforces $\vartheta_{c} \rightarrow 0$. The contact heat flux $h_{o}=-\epsilon_{\theta} \vartheta_{c}$ is pressure independent. This model is central to the micromechanical modeling of macroscopic thermal contact resistance where the continuity of the temperature across the real contact interface, that is, $\vartheta_{c}=0$, is postulated.

(ii) Constitutive model: $\mathcal{H}_{o}=-k_{\theta} p_{N}^{\alpha} \vartheta_{c}^{2} / 2$.

The contact heat flux is $h_{o}=-k_{\theta} p_{N}^{\alpha} \vartheta_{c}$, where $\left\{k_{\theta}, \alpha\right\}$ are material constants with $\alpha$ typically of order $\mathcal{O}(1)$. This model reflects a microscopically non-conforming contact interface due to roughness.

The choice of modeling based on $h_{o}$ versus $h$ has no consequence for the constraint model, both delivering the same asymptotic behavior as $\epsilon_{\theta} \rightarrow \infty$. This is also true for the constitutive model at 
infinitesimal deformations. In the scope of this work, on the other hand, finite deformations are of concern. In this context, modeling with $h_{o}$ is a choice made on convenience and could be extended to match formulations that are expressed in the spatial configuration. As a special case, it is noted that because $p_{N}>0$ is the referential pressure, for the case of $\alpha=1$, one observes

$$
\delta \mathcal{G}_{\theta}^{c}=\int_{\Gamma_{o}^{c}} \delta \vartheta_{c} \underbrace{\left(-k_{\theta} p_{N} \vartheta_{c}\right)}_{=h_{o}} \mathrm{~d} A=\int_{\Gamma^{c}} \delta \vartheta_{c} \underbrace{\left(-k_{\theta} t_{N} \vartheta_{c}\right)}_{=h} \mathrm{~d} a,
$$

thereby capturing the spatial counterpart $\mathcal{H}=-k_{\theta} t_{N} \vartheta_{c}^{2}$ of the dissipation potential with $t_{N}>0$ as the spatial pressure-see Appendix A.

\subsection{Mortar discretization}

The constraint model, in which $\mathcal{H}_{o}$ is a classical penalty potential, suggests the need for a robust framework for enforcing $\vartheta_{c}=0$. Such a framework is offered by mortar-based methods. The presentation is brief, as the structure of the problem condenses to that of normal contact for the constraint model, yet necessary in order to clarify various possible choices. A variational basis for the derivation of the framework is outlined in Appendix B.

A mortar variable $\gamma_{\theta}$ is introduced, and together with the contact heat flux, it is assigned the discretization of the slave surface as in $[37,38]$ :

$$
\gamma_{\theta}=\sum_{I} N^{I} \gamma_{\theta}^{I}, \quad h_{o}=\sum_{I} N^{I} h_{o}^{I} .
$$

Here, the sum is over all the points associated with the shape functions $N^{I}$. These points could be nodes, as in classical Lagrange basis functions, or control points in the context of isogeometric analysis. By using the notation $\langle\bullet\rangle=\int_{\Gamma_{o}^{c}} \bullet \mathrm{d} A$, the overlap matrix components $\Phi^{I J}=\left\langle N^{I} N^{J}\right\rangle$ may be defined. The interface dissipation potential is evaluated using the mortar variable $\gamma_{\theta}$ for variational consistency, that is, $\mathcal{H}_{o}=\mathcal{H}_{o}\left(\gamma_{\theta}, p_{N}\right)$-see Appendix B, such that

$$
\left\langle N^{I} \gamma_{\theta}\right\rangle=\left\langle N^{I} \vartheta_{c}\right\rangle, \quad\left\langle N^{I} h_{o}\right\rangle=\left\langle N^{I} \frac{\partial \mathcal{H}_{o}}{\partial \gamma_{\theta}}\right\rangle
$$

then deliver the discrete variables

$$
\gamma_{\theta}^{I}=\sum_{J} \hat{\Phi}^{I J}\left\langle N^{J} \vartheta_{c}\right\rangle, \quad h_{o}^{I}=\sum_{J} \hat{\Phi}^{I J}\left\langle N^{J} \frac{\partial \mathcal{H}_{o}}{\partial \gamma_{\theta}}\right\rangle
$$

where $\hat{\Phi}^{I J}$ are the components of the inverse of the overlap matrix. In the particular case of a constraint model, it is observed that

$$
h_{o}^{I}=-\sum_{J} \hat{\Phi}^{I J}\left\langle N^{J} \epsilon_{\theta} \gamma_{\theta}\right\rangle=-\sum_{J} \sum_{K} \hat{\Phi}^{I J} \Phi^{J K} \epsilon_{\theta} \gamma_{c}^{K}=-\epsilon_{\theta} \gamma_{\theta}^{I} .
$$

On the other hand, having to compute the inverse of the overlap matrix is unfavorable. Row-sum lumping diagonalizes the matrix such that one obtains the simplified formulation

$$
\gamma_{\theta}^{I}=\left\langle N^{I} \vartheta_{c}\right\rangle /\left\langle N^{I}\right\rangle
$$

This is introduced directly into the form of $h_{o}^{I}$ for the constraint formulation as derived earlier. For the constitutive model, $h_{o}^{I}$ are again recovered by lumping. Because $\left\langle N^{I}\right\rangle>0$ is guaranteed for NURBS, this framework is uniformly applicable to all orders of isogeometric discretizations. Earlier, it was assumed that all slave points are in contact. Incorporating an active set indicator in the formulation requires some care but is standard.

The constitutive formulation of $\mathcal{H}_{o}$ typically delivers a temperature jump that can be significantly different from zero. However, in regions of very high pressure, the jump may be driven toward the constraint limit. This is the motivation for pursuing an approach that is common to both formulations 
of $\mathcal{H}_{o}$. If it is determined that the solution will be far from the constraint limit, a more straightforward formulation that is significantly easier to implement may be pursued by directly using the continuum formulation, for example, (4.3), without a mortar approach. Both choices are expected to deliver identical results away from the constraint limit. In the constraint limit, however, it will be demonstrated that the mortar approach delivers superior numerical performance. For this reason, it is additionally advantageous to enforce the constraint of a vanishing temperature jump as efficiently and accurately as possible. This is discussed next.

\subsection{Constraint enforcement with the augmented Lagrangian approach}

As $\epsilon_{\theta} \rightarrow \infty$ in the constraint model, the mortar variables $\gamma_{\theta}^{I}$ are driven to zero. Section 4.2 enforces this limit through a penalty approach. Within a thermomechanical problem, the magnitude of the heat flux may vary significantly, and therefore, it is difficult to ensure that $\epsilon_{\theta}$ is sufficiently large. To ensure $\gamma_{\theta}^{I} \rightarrow 0$ independent of $\epsilon_{\theta}$, the penalty formulation may be complemented by Uzawa iterations, as is often performed in mechanical contact [40,41]. This framework corresponds to a staggered multiplier update form of the augmented Lagrangian framework. The disadvantage of this approach is that the number of Uzawa iterations needed to reach a desired tolerance on the multiplier convergence depends on a number of parameters, including the discretization, and may be significantly large. For this reason, the original augmented Lagrangian approach will be made use of in this work [45]. It is remarked that the same approach is presently also used for normal contact within a mortar setting, following [46] and others. In the lumped form, a mechanical formulation that makes use of the advantages offered by NURBS has been presented in [28].

In the mortar-based augmented Lagrangian framework, the discrete heat flux is represented as

$$
h_{o}^{I}=\lambda_{\theta}^{I}-\epsilon_{\theta} \gamma_{\theta}^{I}
$$

and is non-zero only if $I$ belongs to the contact active set $\mathcal{A}$. Associated with the Lagrange multipliers $\lambda_{\theta}^{I}$ are the additional equations that augment or deactivate them depending on whether the point remains in contact or loses contact. These equations constitute a variational form $\delta \mathcal{L}_{\theta}^{c}$, which enforces $\gamma_{\theta}^{I}=0$ where there is contact:

$$
\delta \mathcal{L}_{\theta}^{c}=\sum_{I} \delta \lambda_{\theta}^{I}\left\langle N^{I}\right\rangle \Lambda_{\theta}^{I}=0 \quad \longrightarrow \quad \Lambda_{\theta}^{I}= \begin{cases}\gamma_{\theta}^{I} & \text { if } I \in \mathcal{A}, \\ \lambda_{\theta}^{I} / \epsilon_{\theta} & \text { otherwise }\end{cases}
$$

For both the present constraint formulation and the constitutive formulation, the coupled mechanical and thermal contributions are linearized consistently within an iterative Newton-Raphson framework that guarantees asymptotically quadratic convergence when $\mathcal{A}$ does not change.

\section{NUMERICAL INVESTIGATIONS}

In this section, various aspects of the homogenization approach will be demonstrated. The investigations start with the analysis of an asperity contact problem in the context of the thermomechanical extension to the classical Hertzian contact setup. This is followed by a patch test study on flat and curved interfaces, which is an important assessment of the contact algorithm but is also relevant for the analysis of perfectly conforming interfaces. Overall, these two examples motivate the use of a mortar approach through the demonstration of a quantitatively and qualitatively better solution in comparison with a formulation where the thermal contact constraints or constitutive formulations are directly applied pointwise throughout the interface. This latter alternative will be referred to as the continuum approach. Presently, this continuum approach also constitutes the only alternative to mortar-based algorithms because there is no straightforward extension of the classical node-to-surface type formulations to isogeometric discretizations. In all examples, an augmented Lagrangian-based mortar method is used to model frictionless mechanical contact. Therefore, only the penalty and constitutive parameters employed for thermal contact will be explicitly denoted. The penalty parameter $\epsilon_{\theta}$ reported is the base value. It is divided by the length scale inherent to the problem, which is $10^{-5}$ in the numerical investigations. 
The examples are based on finite thermoelasticity as the underlying bulk constitutive formulation. Additionally, a temperature-dependent thermal conductivity $k$ is employed within the micromechanical tests such that

$$
k=k_{o}+k_{1}\left(\theta-\theta_{o}\right),
$$

where $\theta_{o}$ denotes the initial equilibrium temperature of the medium. An exception is the Hertzian contact example where the linearized formulation was employed with a constant conductivity $k_{o}$. Some of the fundamental material parameters employed are summarized in Table I. The remaining aspects [33] of the employed finite thermoelasticity model are omitted—see also Appendix A.

The numerical discretization follows isogeometric analysis through the use of second-order NURBS basis functions unless otherwise noted, with four/eight Gauss-Legendre quadrature points per spatial direction of the bulk/interface. For rough surfaces, the RMS roughness will be explicitly denoted where necessary. In most investigations, sinusoidal roughness with a period of $10 \mu \mathrm{m}$ and an RMS of $1 \mu \mathrm{m}$ will be employed. Both bodies are assigned the same height $H_{o}$. Finally, the macroscopic algorithmic designation of master will be reflected onto the lower body so that the superscript on $\bar{\vartheta}_{c}$ will not be explicitly denoted unless necessary. Consequently, $\partial \mathcal{C}_{o}^{(2), e}$ (Figures 1 and 2) will be kept at a fixed temperature and displacement, whereas $\left\{\bar{p}_{N}, \bar{h}_{o}\right\}$ are applied uniformly on $\partial \mathcal{C}_{o}^{(1), e}$ (Figure 5). Because macroscopic in-plane deformation is not imposed, $\bar{t}_{N}=\bar{p}_{N}$ and $\bar{h}=\bar{h}_{o}$.

\subsection{Asperity contact: singular solutions}

Contact on the microscale is dominated by the interaction between the asperities of the two rough surfaces. Hence, it is of fundamental importance to be able to accurately model contact between two asperities. This situation is idealized in the context of the thermoelastic extension to the classical Hertzian contact problem in a plane strain setting. The geometry of the problem is described

Table I. Major simulation parameters employed are summarized.

\begin{tabular}{lccc}
\hline Parameter & Unit & Symbol & Value \\
\hline Young's modulus & $\mathrm{N} / \mathrm{mm}^{2}$ & $E$ & 1.0 \\
Poisson's ratio & $\mathrm{N} / \mathrm{mm}^{2}$ & $v$ & 0.3 \\
Linear thermal expansion coefficient & $\mathrm{K}^{-1}$ & $\alpha_{o}$ & $10^{-5}$ \\
Thermal conductivity & $\mathrm{W} / \mathrm{mK}^{2}$ & $k_{o}$ & 1 \\
& $\mathrm{~W} / \mathrm{mK}^{2}$ & $k_{1}$ & 0.01 \\
Initial temperature of the medium & $\mathrm{K}$ & $\theta_{o}$ & 293.15 \\
Macroscopic heat flux (from $\mathcal{B}^{(1)}$ to $\left.\mathcal{B}^{(2)}\right)$ & $\mathrm{W} / \mathrm{mm}^{2}$ & $\left|\bar{h}_{o}\right|$ & 1.0 \\
\hline
\end{tabular}

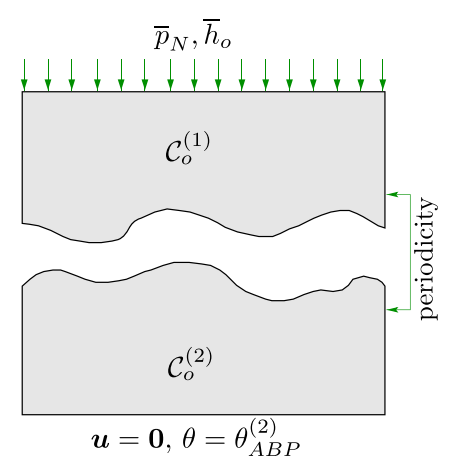

Micromechanical Tests

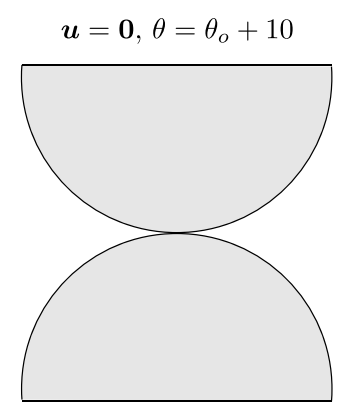

$\boldsymbol{u}=\mathbf{0}, \theta=\theta_{o}$

AsPERITy CONTACT

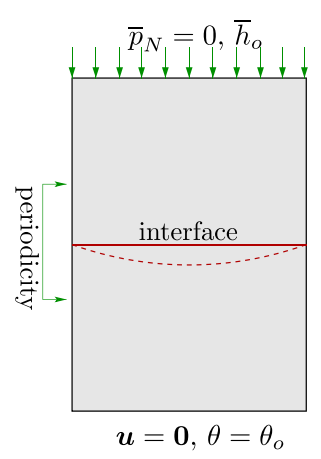

PATCh TESTS

Figure 5. The boundary conditions employed in the numerical investigations are summarized in a two-dimensional setting. Here, $\boldsymbol{u}$ is the displacement. For the micromechanical tests, see also Figure 2. 
in [32] along with the three choices of discretization, namely coarse/intermediate/fine, and therefore is not repeated. All solutions are based on the coarse discretization except for the study on third-order NURBS discretizations. The deviation from this classical purely mechanical setting is as follows. The top and bottom cylindrical bodies are kept fixed under displacement control. Simultaneously, the temperature at the non-contact portion of the upper body is elevated by $10 \mathrm{~K}$, whereas its counterpart for the lower body is kept at the initial temperature. The bodies expand and come into contact (Figure 5). The solutions were obtained in a coupled thermomechanical framework. The induced pressure distribution follows the classical Hertz solution so that the numerical trends previously reported in [33] for the purely mechanical case hold again. By concentrating on the thermal aspects, the accompanying normal heat flux distribution was obtained by [47]

$$
h_{o}=\frac{h_{o}^{\min }}{\sqrt{1-(r / a)^{2}}} .
$$

Here, $r$ is the distance to the center of the contact interface with width $2 a$ and

$$
h_{o}^{\min }=\frac{Q}{\pi a},
$$

with $Q$ as the total heat flux through the interface. This solution is based on the assumption of a constraint model for the dissipation potential $\mathcal{H}_{o}$. In the figures, the non-dimensional parameters $h_{o}{ }^{\prime}=h_{o} / h_{o}^{\min }$ and $r^{\prime}=r / a$ are employed.

The analytical solution displays the singularity that is inherent to the thermal problem, unlike the mechanical problem. The development of this singularity, as the parameter $k_{\theta}$ of the constitutive model is increased, is demonstrated in Figure 6 for the mortar method and compared with the analytical solution for the constraint limit $k_{\theta} \rightarrow \infty$. A smooth solution is obtained for $k_{\theta}=1$ with no visible singularity development. A solution that is closer to the constraint limit is observed for $k_{\theta}=10^{2}$. For both cases, an agreement between the mortar and the continuum solutions is observed. In [48], solutions are presented for a range of constitutive models where the gradual development of such singularities toward the constraint limit is analyzed. Finally, the constraint model is solved on the basis of the augmented Lagrangian approach. The singularity places stringent requirements on the numerical method. The solution accurately matches the analytical one in the middle of the contact zone; however, oscillations are induced toward the edges owing to the inability of the finite element solution to capture the singularity. Similar problems have been observed in the context of the purely mechanical Hertz problem, although much milder owing to the absence of a singularity, in which case they have been attributed to the use of higher-order basis functions [49]. Such an observation appears to hold for the present case as well, as summarized in Figure 7. Here, third-order NURBS basis functions are employed. In the coarse discretization, the solution is slightly more oscillatory than the corresponding second-order discretization. On the other hand, the quality of the solution rapidly increases with mesh refinement, where an accurate agreement with the analytical solution is observed away from the singular region. These results seem to indicate that linear elements may be more suitable to this class of problems. On the other hand, the results

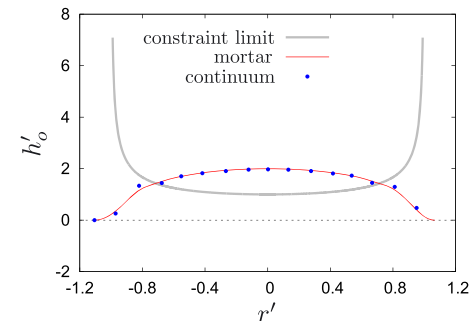

(a) constitutive model $\left(k_{\theta}=1\right)$

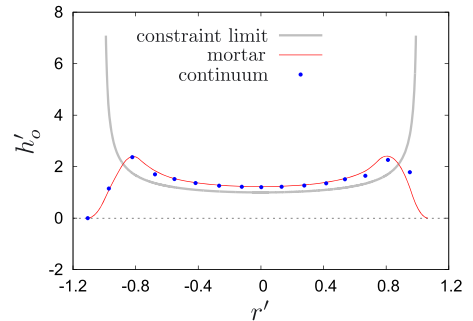

(b) constitutive model $\left(k_{\theta}=10^{2}\right)$

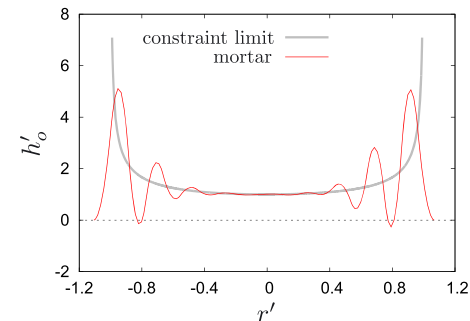

(c) constraint model

Figure 6. The development of the heat flux singularity is demonstrated for the thermoelastic Hertzian contact problem. The constraint model is solved by the augmented Lagrangian algorithm. 


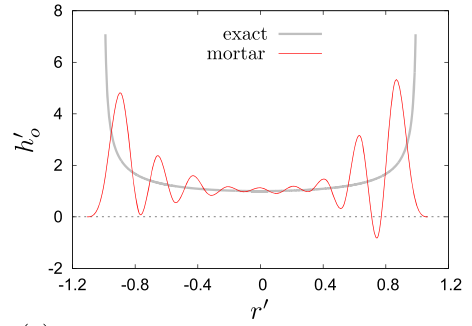

(a) coarse

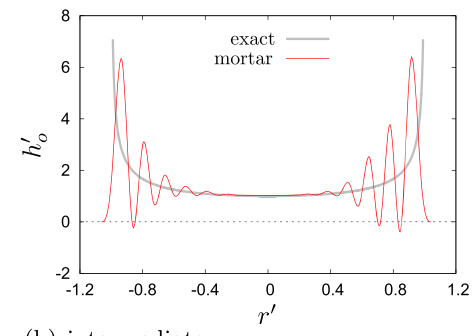

(b) intermediate

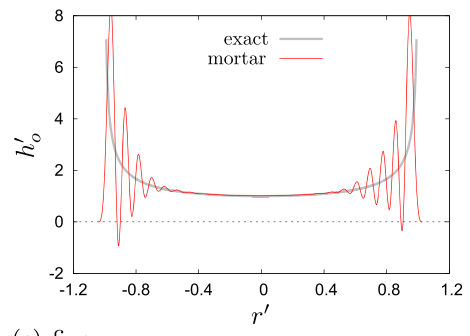

(c) fine

Figure 7. For third-order NURBS basis functions, the effect of discretization is summarized for the thermoelastic Hertzian contact problem with the constraint model—cf. Figure 6.

also demonstrate the uniform applicability of the mortar approach to higher-order NURBS discretizations. In view of the additional advantages that NURBS basis functions offer, particularly in terms of smoothness, second-order NURBS basis functions will be employed throughout the remaining analysis.

Figure 6 shows the equivalence of the continuum approach with the mortar algorithm for the constitutive model at finite temperature jumps. As the parameter $k_{\theta}$ is driven to infinity, the mortar approach develops toward the solution obtained with the augmented Lagrangian algorithm. The continuum approach, on the other hand, delivers an ill-conditioned behavior as summarized in Figure 8. The oscillations induced by the singularities propagate inwards from the edges, causing unacceptable solution quality in the center region as well. Lower values of the penalty parameter are also unacceptable because these deliver a non-negligible temperature jump across the contact interface. Moreover, the continuum approach cannot converge in a single load step for large values of the penalty parameter, thus rendering this approach numerically inefficient compared with the mortar one that converges in a single step for all simulations presented in this section. To summarize, the

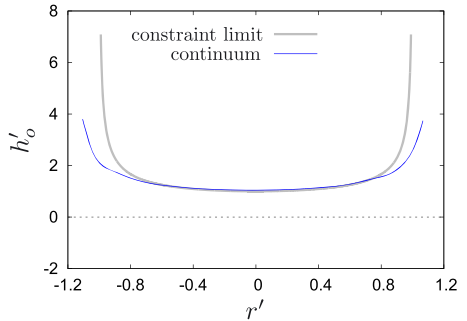

(a-1) constraint model $\left(\epsilon_{\theta}=10\right)$

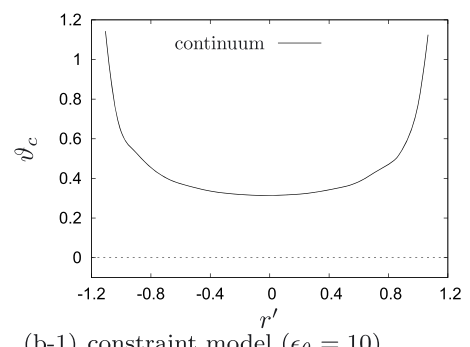

(b-1) constraint model $\left(\epsilon_{\theta}=10\right)$

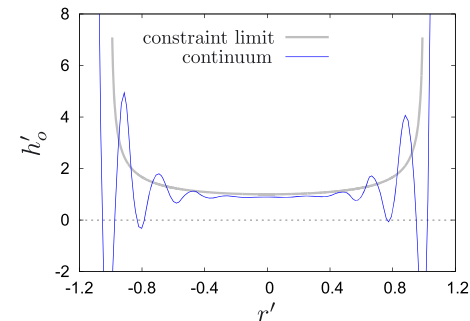

(a-2) constraint model $\left(\epsilon_{\theta}=10^{3}\right)$

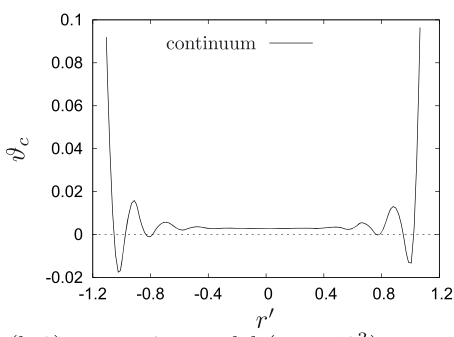

(b-2) constraint model $\left(\epsilon_{\theta}=10^{3}\right)$

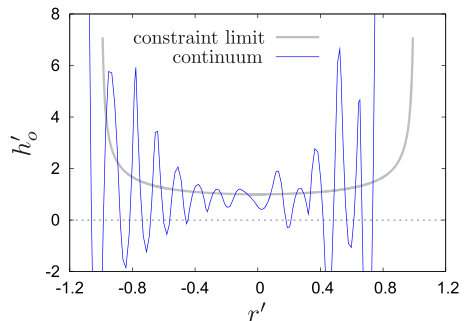

(a-3) constraint model $\left(\epsilon_{\theta}=10^{5}\right)$

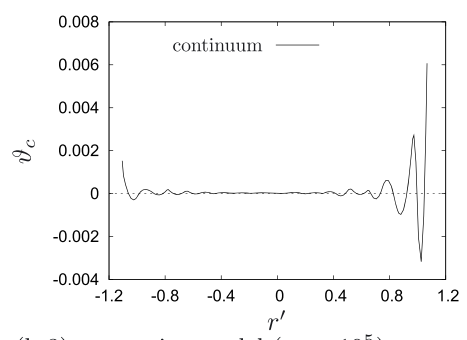

(b-3) constraint model $\left(\epsilon_{\theta}=10^{5}\right)$

Figure 8. The interface heat flux and the temperature jump are summarized for various penalty parameters in the context of the continuum constraint enforcement algorithm, which displays a behavior that is significantly inferior to the mortar results in Figures 6 and 7 near the constraint limit. 
mortar approach delivers a unified treatment of both the constitutive and constraint models where the transition from the former to the latter is captured in a numerically efficient manner.

\subsection{Patch tests}

The thermoelastic Hertzian contact problem of the previous section demonstrated the local quality of the solution provided by the mortar-based method in the context of the constraint model. The present example constitutes a global solution quality demonstrator through three-dimensional patch test studies (Figure 9). Within these tests, two bodies with an edge length of $10 \mu \mathrm{m}$ are held together at zero pressure while a heat flux $\bar{h}_{o}$ is applied in ten load steps (Figure 5). Because of the exact geometry representation by NURBS, the interfaces are perfectly conforming geometrically although the discretizations are clearly non-matching and coarse. When the interface is flat, both the continuum method with $\epsilon_{\theta}=10$ and the mortar method with the augmented Lagrangian algorithm deliver a uniform flux distribution as expected since the bodies should behave as one. When the interface is curved, the bodies should still behave as one, which, indeed, is the case with the mortar method where the temperature jump is exactly enforced to zero. If the augmented Lagrangian algorithm was not employed, the flux would clearly not be uniform. This appears to be reflected in the continuum method solution, where a highly non-uniform flux is obtained although the maximum temperature jump is slightly less than $1 \mathrm{~K}$ across the interface, while the temperature variation from the bottom surface to the top surface is more than $30 \mathrm{~K}$. The major difference, however, is that whereas the mortar method in the penalty setting easily delivers a solution of acceptable quality at $\epsilon_{\theta}=10^{6}$ with a corresponding temperature jump of less than $10^{-6} \mathrm{~K}$ across the interface, the continuum method fails to converge at this value of the penalty parameter. Smaller values of the penalty parameter,

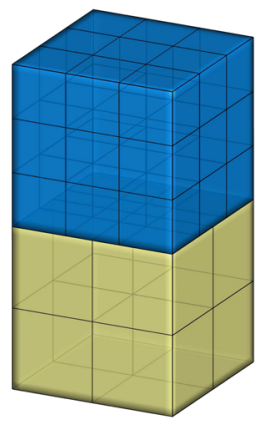

(a-1) flat interface

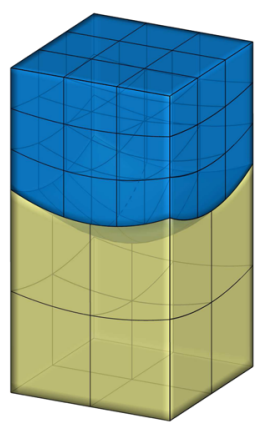

(b-1) curved interface

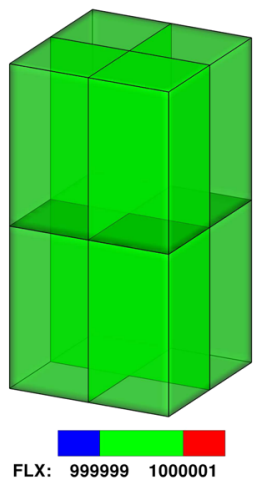

(a-2) continuum $\left(\epsilon_{\theta}=10\right)$

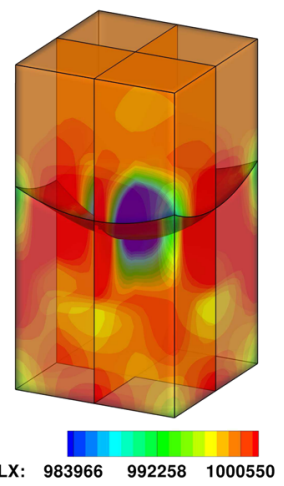

(b-2) continuum $\left(\epsilon_{\theta}=10\right)$

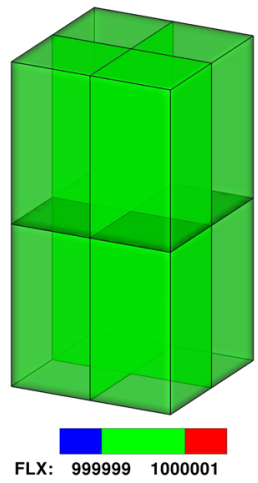

(a-3) mortar

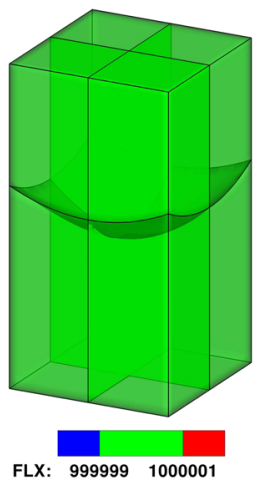

(b-3) mortar

Figure 9. The patch tests as discussed in Section 5.2 are summarized. Here, FLX: $\|\boldsymbol{q}\|$. 
on the other hand, fail to deliver a satisfactory solution. This result is conceivably due to the inferior behavior of the continuum method as earlier demonstrated in Figure 8. Because the interfaces are geometrically perfectly matching and mechanical contact is based on augmented Lagrangian with mortar in all cases, one can safely state that these observations pertain neither to the geometry nor to the mechanical contact algorithm but purely to the method used for thermal contact. In all subsequent examples, the mortar method will be used for thermal contact.

\subsection{Boundary conditions}

The influence of the BCs on the macroscopic response is well-known in the homogenization literature. In this section, this influence is briefly addressed using periodic samples with a sinusoidal surface while assuming the constraint model for the interface dissipation potential. Figure 10 displays a three-dimensional sample with matching surfaces such that the whole interface is in contact. In this case, both PR-BCs and LN-BCs deliver the same result. When the interface is mismatched, LN-BCs predict a gap for a single unit cell with the prescribed height $H_{o}$ so that $\bar{\vartheta}_{c}=6.4 \mathrm{~K}$ is observed. The PR-BCs, on the other hand, predict a slightly smaller gap because they allow the samples to slide over each other freely, leading to a smaller jump $\bar{\vartheta}_{c}=5.8 \mathrm{~K}$. The situation is more easily observed in a two-dimensional setting (Figure 11). As the height of the samples increases, PR-BCs deliver smaller and smaller gaps because of the degree of freedom in lateral motion. This motion essentially corresponds to the macroscopic sliding of the surfaces so that the interface matches exactly. In other words, the exact value of $\bar{\vartheta}_{c}$ for this setup is zero. LN-BCs do not provide this degree of freedom and therefore deliver always the same result for a unit cell of any given height. In order to approach the anticipated result, an increase in height must be accompanied by sample enlargement where more unit cells are involved.

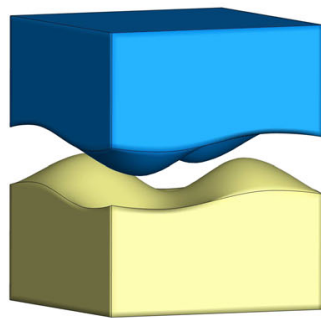

(a) matching

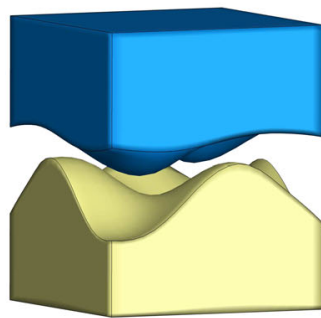

(b) mismatching

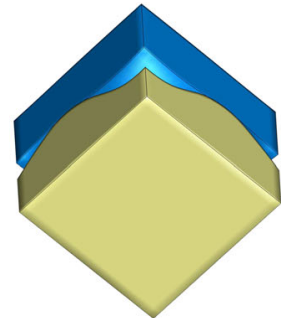

(c) LN-BCs

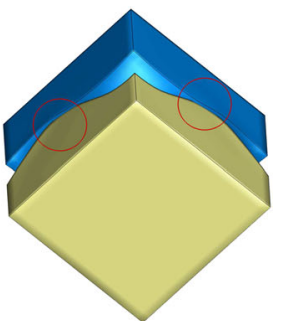

(d) PR-BCs

Figure 10. Sinusoidally rough surfaces in contact: linear (LN) and periodic (PR) boundary conditions (BCs) are compared. Note that PR-BCs allow the samples to slide with respect to each other during contact.

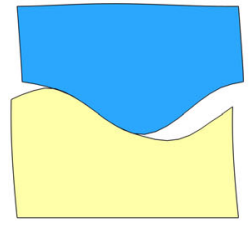

(a) PR-BCs $\left(H_{o}\right)$

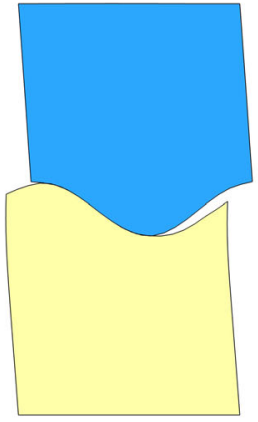

(b) PR-BCs $\left(2 H_{o}\right)$

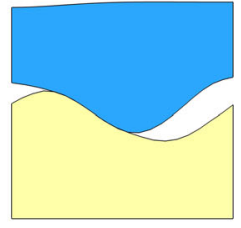

(c) LN-BCs $\left(H_{o}\right)$

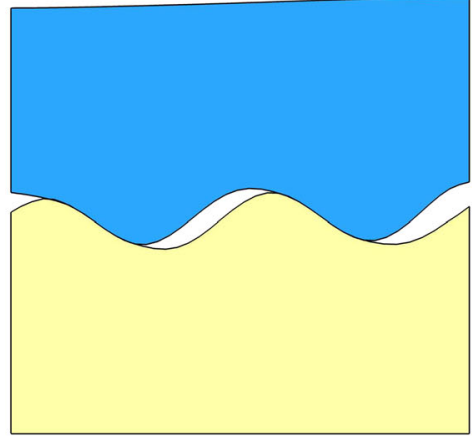

(d) LN-BCs $\left(2 H_{o}\right)$

Figure 11. Sinusoidally rough surfaces in contact: the effects of sample height and width are demonstrated. 
These observations highlight the advantages of PR-BCs. A complication with their use is that they must be complemented by PR-BCs on the contact interface, that is, the portion of the upper body protruding over the lower one must eventually come into contact with the periodic image of the lower body. To avoid this complication, in the majority of the remaining calculations, periodic microstructures will be employed where PR-BCs and LN-BCs will deliver the same results irrespective of the sample size. Consequently, it will be sufficient to use a single unit cell provided the height of the samples, which is always a free variable, is suitably chosen.

\subsection{Textured interfaces}

The analysis of Section 3.2 is numerically investigated in this section. For this purpose, the sinusoidal matching texture of Figure 10 is employed together with the random matching interface texture in Figure 12 where the RMS roughness is $0.5 \mu \mathrm{m}$. The random texture is generated through a random-field model that displays in-plane isotropy as well as out-of-plane Gaussian features [17]. In the present analysis, the interface dissipation potential employs $k_{\theta}=10$ and $\alpha=1$. The twophase homogenization setup is used where self-consistency iterations are not necessary because of interface conformity. Clearly, both periodic and random textures display a macroscopic jump that is less than the jump for a flat interface, thereby verifying the analysis of Section 3.2. Indeed, the two-phase approach delivers the same macroscopic response as that predicted by (3.5). Moreover, the jump decreases further when the texture RMS is increased. Clearly, perfect interface conformity is the key to these observations. Interfaces that slightly mismatch may easily lead to a higher macroscopic resistance.

\subsection{Spurious size effects}

Figure 13 displays several size effects. In the first scenario, the setup of Section 2.2.3 is considered where the macroscopic jump is expected to correspond to the microscopic one, which is $10 \mathrm{~K}$ under the prescribed test conditions. The two-phase framework correctly captures the macroscopic jump, and this value is independent of the sample height. The fully coupled frameworks based on Equations (2.14) and (3.4) fail to capture the macroscopic jump, both significantly deviating from the correct value with increasing sample height. In a second scenario, a sinusoidal surface contacting an initially flat one is considered in the framework of the constraint model, and a macroscopic flux of $\bar{h}_{o}=0.01 \mathrm{~W} / \mathrm{mm}^{2}$ is applied. Here, the discussion of Section 3.1 is demonstrated. The scale $\varepsilon$ denotes the initial width of the sample. Beyond a certain limit, it is expected that $\bar{\vartheta}_{c} \rightarrow 0$ such that the measured jump $\bar{\vartheta}_{c}$ scales linearly with $\varepsilon$. For all sample sizes, the two-phase framework without self-consistency iterations retains this linear relation since the scale separation limit was the motivation behind the framework. Moreover, because the self-consistency iterations are omitted, the

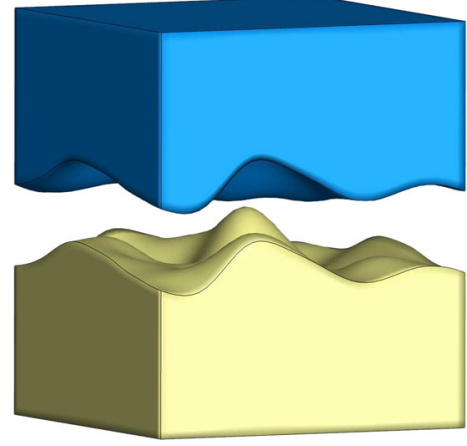

(a) random interface texture

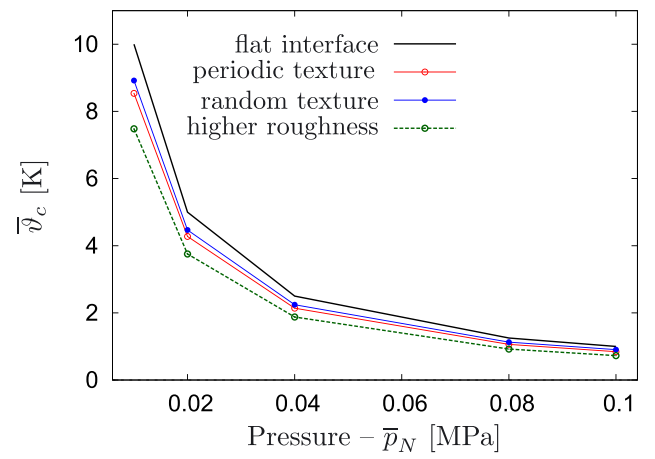

(b) dissipation reduction

Figure 12. The analysis of Section 3.2 is demonstrated numerically. The higher roughness results correspond to the periodic case with a root-mean-square roughness of $1.5 \mu \mathrm{m}$. 


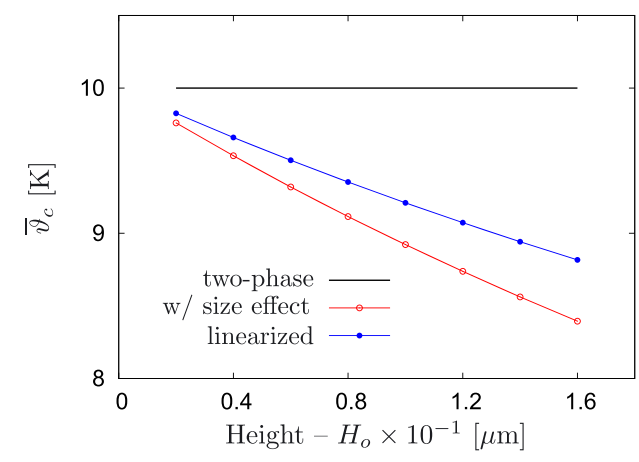

(a) sample height is varied

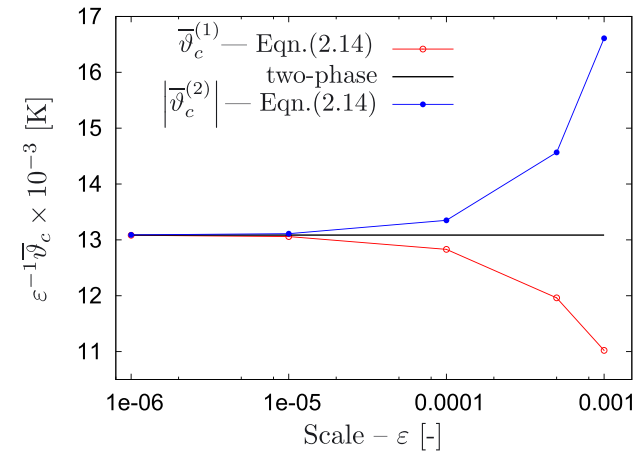

(b) sample dimensions are scaled

Figure 13. (a) A flat, resistive interface is chosen such that the microscopic temperature jump is 10, which is correctly captured on the macroscale by the two-phase framework only. (b) A sinusoidal surface contacts an initially flat one in the constraint setting. $\bar{\vartheta}_{c}(I)$ are based on the coupled result (2.14).

results are independent of which surface is chosen as master/slave. The result (2.14) clearly displays size effects and additionally reflects the effect of the master/slave choice, both of which disappear at scale separation only.

\subsection{Self-consistency and sample height}

Self-consistency iterations in the two-phase framework typically converge rapidly, as summarized in Figure 14. In particular, the iterations converge faster when $\bar{\vartheta}_{c}$ is smaller. Additionally, the effect of the sample height, which was also discussed in Section 5.5 in the context of resistive interfaces, should be investigated. The two-phase framework with self-consistency iterations delivers a macroscopic response that is virtually independent of the sample height for samples that are sufficiently high. This response is compared with the results obtained using the coupled framework and equation (2.14) as well as with its linearized counterpart (3.4). Additionally, the surface average of the temperature jump throughout the whole interface, including the non-contact portion, is plotted. Because the interface is rough, the average is conducted over the slave surface by projecting the value of the jump to the mean plane of either surface. It is observed that this value is also independent of the sample height but is different than the two-phase framework result that will be shown to deliver a more accurate homogenized response in the next section. Finally, the coupled frameworks deliver height-dependent results that improve in quality as the height decreases. Although it seems that

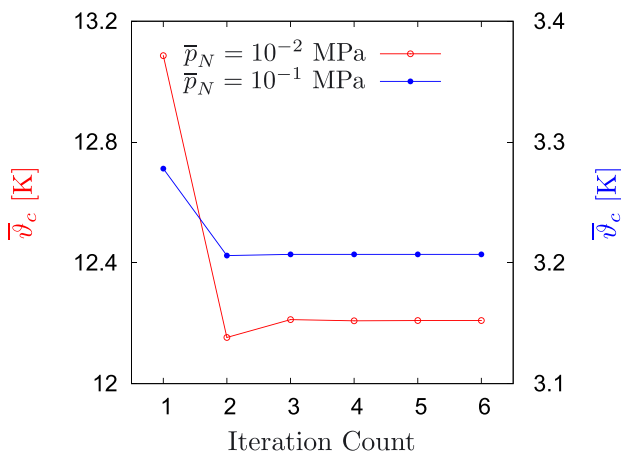

(a) self-consistency

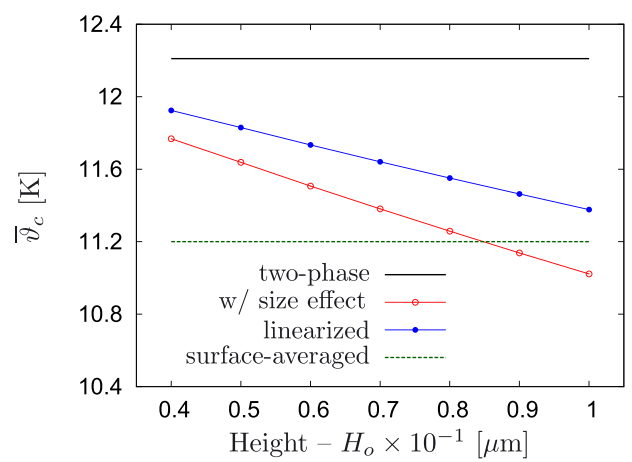

(b) size effects

Figure 14. (a) Convergence of self-consistency iterations is demonstrated for the two-phase framework based on the constraint model for the contact interface. (b) The effect of the sample height is shown for various methods of characterizing $\bar{\vartheta}_{c}$. 
driving the height to zero is an appropriate approach, similar to the resistive interface example of Figure 13, this is not possible with rough interfaces as shown in Figure 15, in this case with a coupled analysis. Unless the sample height is an appropriately large value, for example, more than $2 \mu \mathrm{m}$, the contact interface is unrealistic. As the height increases, both the deformation and the temperature distribution become independent of the height in the vicinity of the contact interface. Additionally, the same solution is obtained irrespective of the surface on which the pressure is applied. All of these observations indicate the necessity for a framework that delivers a saturated response with increasing sample height. The two-phase framework with self-consistency appears to be one such framework.

\subsection{Pressure dependence and validation}

Independent of whether microscale contact resistance exists $\left(\vartheta_{c} \neq 0\right)$ or not $\left(\vartheta_{c}=0\right)$, the macroscopic response represented by $\bar{\vartheta}_{c}$ displays pressure dependence. This dependence is exemplified by the results summarized in Figure 16 where the sinusoidal-on-flat setup of Figure 15 is employed. For the case with microscale contact resistance, the homogenization framework was extended along the discussion of Section 2.2.2. In all computations, the sample heights were chosen to be $7 \mu \mathrm{m}$, whereas the width and the RMS roughness remain at the previously chosen values (10 and $1 \mu \mathrm{m}$, respectively). Because increasing pressure drives the jump to zero, the results with alternative approaches match closely at high pressures. However, the deviation of alternative methods from the two-phase self-consistent framework is observed to be more than $10 \%$ at low pressures. Moreover, it should be recalled that the sample height choice of $7 \mu \mathrm{m}$ remains arbitrary for these alternative methods because no clear asymptotic response was observed in Figure 14 with varying
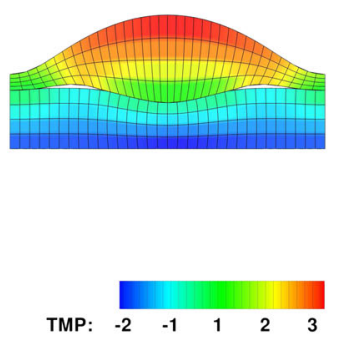

(a-1) $H_{o}=2 \mu \mathrm{m}$
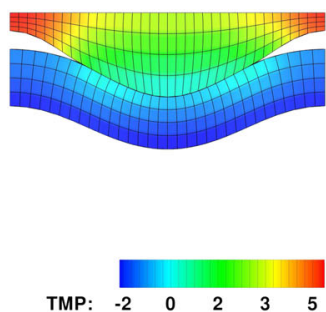

(b-1) $H_{o}=2 \mu \mathrm{m}$
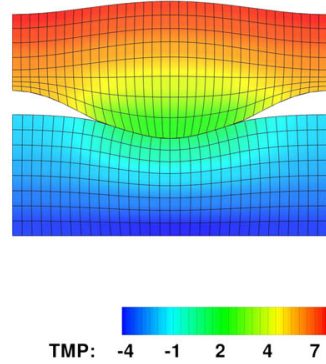

(a-2) $H_{o}=4 \mu \mathrm{m}$
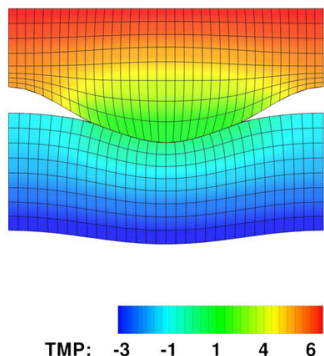

(b-2) $H_{o}=4 \mu \mathrm{m}$

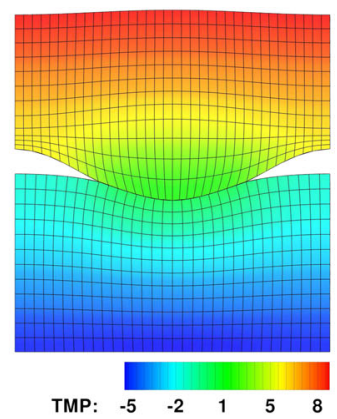

(a-3) $H_{o}=6 \mu \mathrm{m}$

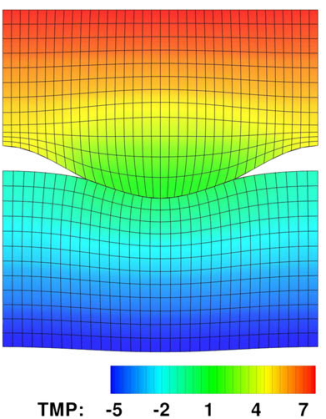

(b-3) $H_{o}=6 \mu \mathrm{m}$

Figure 15. The effect of the sample height on the deformation and temperature fields in the vicinity of the contact interface is shown: (a) the pressure is applied at the top surface while the bottom surface is held fixed, (b) the ordering is switched. Here, TMP: $\theta-\theta_{o}$. 

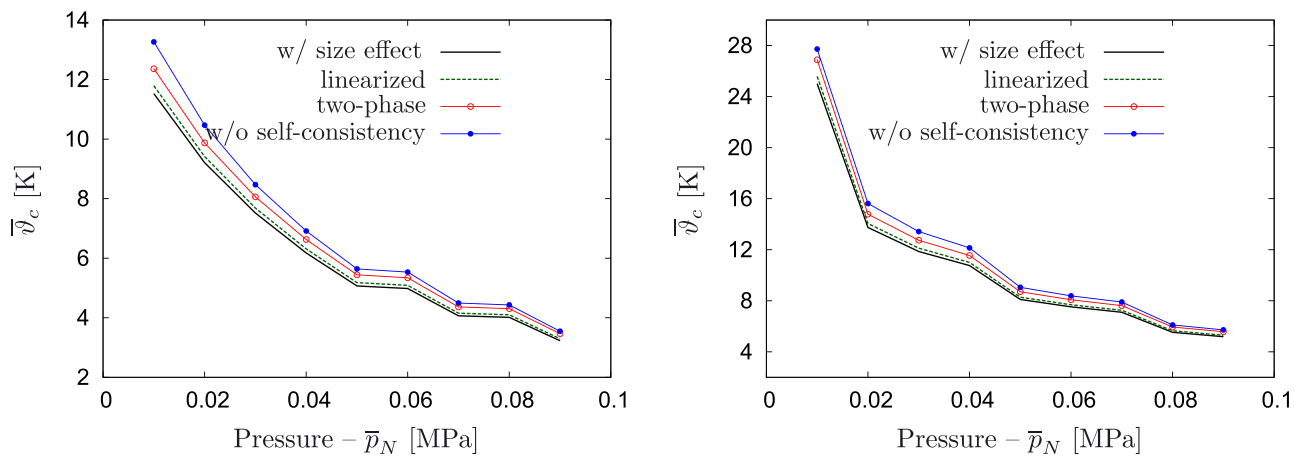

(a-1) $\vartheta_{c}=0$

$(\mathrm{a}-2) \vartheta_{c} \neq 0$
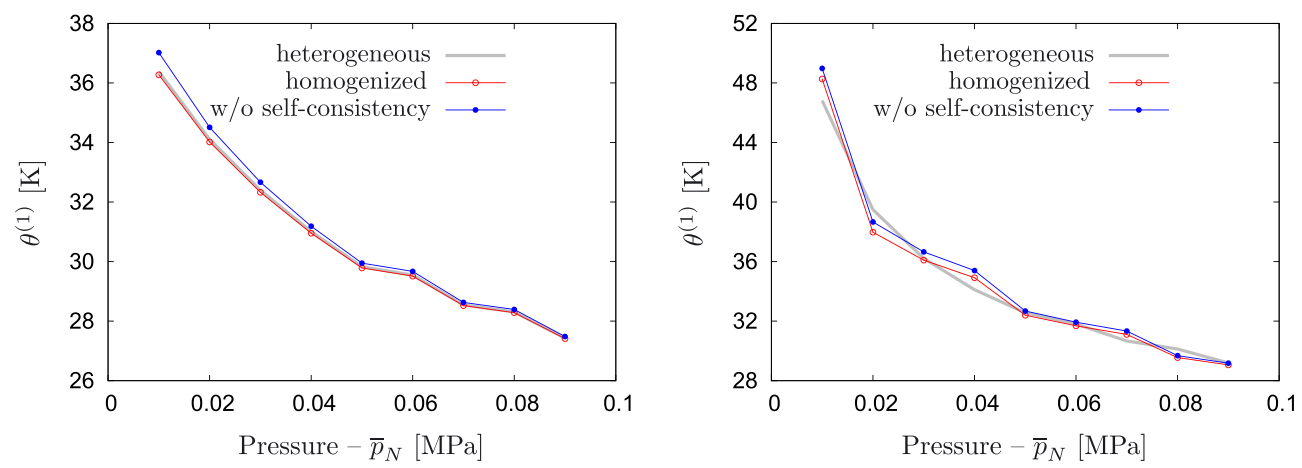

(b-1) $\vartheta_{c}=0$

$(\mathrm{b}-2) \vartheta_{c} \neq 0$

Figure 16. (a) Pressure dependence of $\bar{\vartheta}_{c}$ is demonstrated both for the constraint model $\left(\vartheta_{c}=0\right)$ and for the presence of microscale contact resistance $\left(\vartheta_{c} \neq 0\right)$. (b) For validation, the homogenization predictions with and without self-consistency corrections are compared with the original heterogeneous problem via the temperatures measured at the top of the original heterogeneous and homogenized bar problems of Section 2.2.2.

sample heights. Additionally, the validation of the advocated framework further supports its advantages. The validation is carried out by solving the OHP of Figure 2 in a fully coupled setting. The height for this purpose is chosen to be relatively high $(30 \mu \mathrm{m})$ so that the computational difficulty of dealing with such heterogeneous problems partially appears, namely that the interface must be numerically resolved together with the remaining portion of the domain in order to obtain a reliable value for the monitored quantity. In this case, the monitored quantity is the temperature $\theta^{(1)}$ at the top surface of the upper body. Simultaneously, the homogenized bar problem is solved by enforcing the jump that was computed and the temperature $\theta_{\mathrm{HBP}}^{(1)}$ at the top surface of the upper body is recorded. As also indicated in Figure 2, one expects $\theta_{\mathrm{HBP}}^{(1)}=\theta^{(1)}$ if the homogenization framework is accurate. In Figure 16 for the constraint model, it is observed that the two-phase framework without self-consistency is close to the heterogeneous result and the framework prediction is very accurate after self-consistency. The mismatch without self-consistency is expected because it was motivated by the scale separation limit. The higher accuracy with self-consistency demonstrates the ability of the first-order corrector to take into account finite size effects appropriately. However, the framework remains approximate even after the correction. This is reflected in the case when there is microscale contact resistance and the macroscopic jump is higher. In this case, finite temperature changes dominate the response, and self-consistency does not act as an equally accurate corrector, suggesting possibilities for further improvement.

Finally, it is noted that mortar-based frameworks are expected to deliver a contact response that varies smoothly with an evolving contact interface. This was advocated as a primary strength of 
these approaches in the initial works [35,36], provided that mortar integrals were evaluated accurately using an intermediate surface. From this point of view, the non-smooth variations in the pressure-dependent behavior, which eventually reflect to the validation problem, are unsatisfactory to a certain extent. This non-smoothness was observed to decrease with mesh refinement. Because an intermediate integration surface was not employed in the present work, this is one potential source for this observation. Nevertheless, no degradation was observed in the numerical reliability.

\subsection{Effects of randomness}

Various three-dimensional computations were presented in the preceding discussions. In particular, the depiction of the self-consistent two-phase framework in Figure 4 is based on a computation where randomly rough surfaces were employed. With such random contact interface topographies, the classical homogenization approach requires the determination of a statistically representative sample based on the procedures of ensemble averaging combined with sample enlargement, which lead to a rapidly increasing computational cost. To highlight the effects of randomness, a two-dimensional setup is considered with one-sided as well as two-sided (non-matching) random roughness. The results are summarized in Figure 17. For small sample sizes, the pressure dependence varies significantly with the choice of the sample. For a given pressure, on the other hand, the scatter in the responses from 10 different samples decreases with increasing sample size. The results indicate that it may be necessary to consider even larger sample sizes for a statistically representative macroscopic response. This sample enlargement procedure is manageable in two dimensions (Figure 18) but remains challenging in three dimensions.

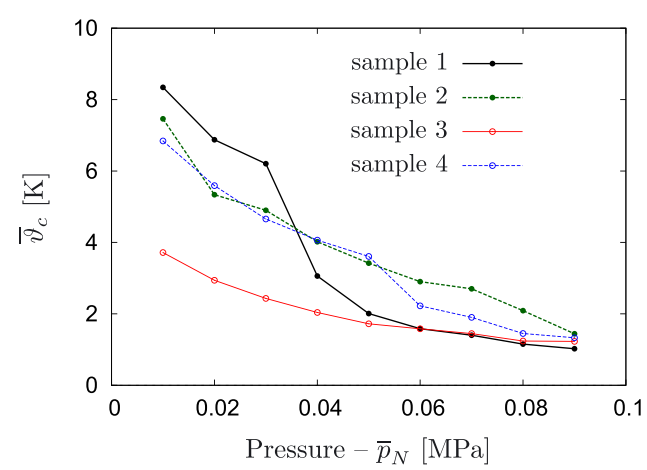

(a-1) one-sided roughness $($ size $=1)$

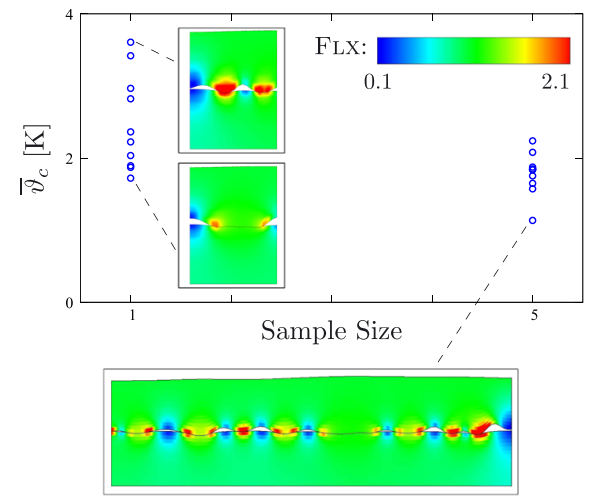

(b-1) one-sided roughness $\left(\bar{p}_{N}=0.05\right)$

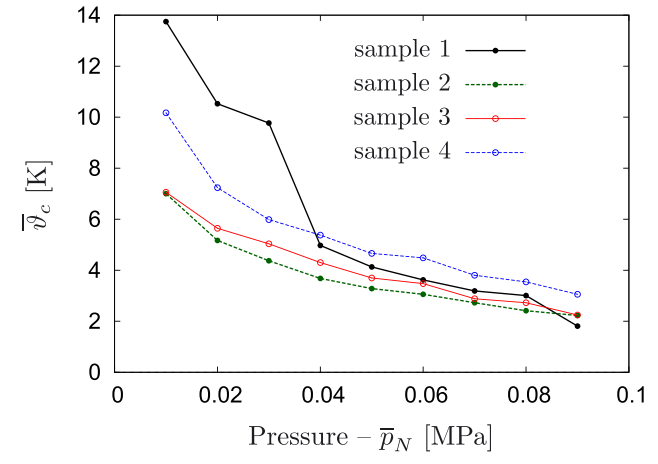

(a-2) two-sided roughness $($ size $=1)$

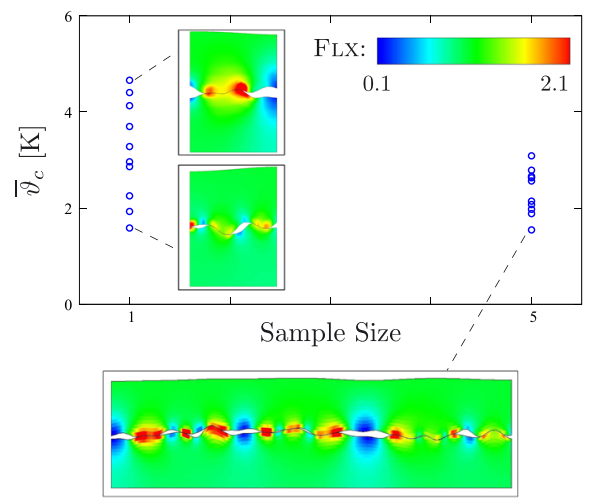

(b-2) two-sided roughness $\left(\bar{p}_{N}=0.05\right)$

Figure 17. The effect of randomness is demonstrated. The qualitative effect appears already without selfconsistency which, therefore, is not employed. The size indicator scales the width of the sample, with 1 corresponding to the default width $(10 \mu \mathrm{m})$. FLX denotes the magnitude of the heat flux in $\mathrm{W} / \mathrm{mm}^{2}$.

Two-sided roughness employs the same root-mean-square roughness $(0.5 \mu \mathrm{m})$ on both surfaces. 

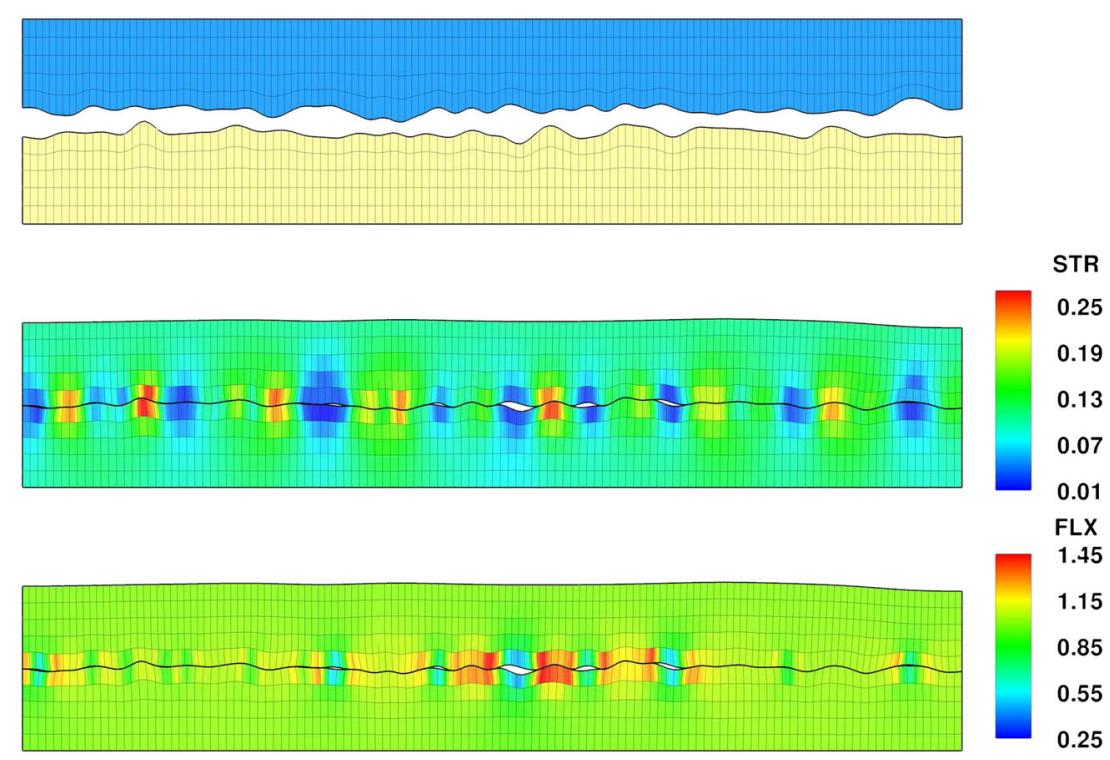

Figure 18. A two-dimensional random interface topography computation. The width of the samples is $100 \mu \mathrm{m}$ whereas the height is $10 \mu \mathrm{m}$ and the root-mean-square roughness is $0.5 \mu \mathrm{m}$. The computation is thermomechanically fully coupled. Along with the original undeformed samples (top), the magnitude STR of the Cauchy stress in MPa (middle) and the magnitude FLX of the heat flux in $\mathrm{W} / \mathrm{mm}^{2}$ (bottom) are shown.

\section{CONCLUSION}

The major advantage of computational homogenization techniques over analytical ones is their ability to incorporate the exact topology and the material behavior of the microstructure, to within modeling approximations, into the analysis framework. In this work, a computational homogenization framework was developed in the context of the thermomechanical contact of two boundary layers with microscopically rough surfaces. The major goal was to accurately capture the macroscopic thermal contact response, which reflects as a temperature jump, and at the same time obtain a homogenized behavior that is independent of algorithmic choices such as master/slave surface designation within the macroscale contact formulation. The framework that was developed for this purpose was motivated by the limit of scale separation such that an algorithmically consistent twophase computational test was induced: a purely mechanical analysis followed by a purely thermal one. However, the homogenization problem becomes practically irrelevant as the scale separation is enforced in the classical asymptotic sense because the macroscopic temperature jump vanishes whenever contacting points pick the same temperature on the microscale. In order to correctly take into account finite size effects that are inherent to the problem, the two-phase framework was cast within a self-consistent iterative scheme that acts as a first-order corrector. Numerical investigations demonstrated various aspects of the proposed homogenization framework and compared it with alternative approaches as well as with the solution of the original heterogeneous problem for purposes of validation. It should be highlighted that while the two-phase framework decouples the mechanical and thermal fields on the microscale, the homogenized thermomechanical contact problem on the macroscale is always coupled. Specifically, a coupled problem must be solved for validation and also for comparison with coupled homogenization frameworks. For this purpose, and in order to offer an alternative to recently proposed mortar-based thermomechanical contact algorithms, an isogeometric approach that was motivated by a classical three-field mixed variational formulation was additionally presented. Overall, the two-phase framework combined with the mortar-based contact algorithm delivers a computationally efficient framework.

The present approach emphasized finite deformations and finite temperature changes in the context of a two-body contact problem. A more realistic setup for the analysis of TIMs is a three-body contact problem where a layer of a finitely deformable material is compressed between 
two other relatively stiff metallic surfaces. Large plastic deformations near the contacting asperities of such metallic surfaces, the modeling of which requires employing small-scale models such as crystal plasticity, also call for a finite deformation framework even without an interface material. A detailed analysis of such problems in a three-dimensional setting with random interface topographies is left for future investigation.

\section{ACKNOWLEDGEMENTS}

Support for this work was provided by the Scientific and Technological Research Council of Turkey (TÜBITAK) under the Career Programme Grant No. 110M661. The author would like to thank C. Hesch for the helpful discussions on mortar-based contact algorithms.

\section{APPENDIX A: CONTINUUM FRAMEWORK}

The reference (current) configuration $\mathcal{R}_{o}(\mathcal{R})$ occupied by a body is assigned a position vector $\boldsymbol{X}$ $(\boldsymbol{x})$, outward unit normal vector $\boldsymbol{N}(\boldsymbol{n})$ on the boundary $\partial \mathcal{R}_{o}(\partial \mathcal{R})$, infinitesimal volume $\mathrm{d} V(\mathrm{~d} v)$, infinitesimal surface area $\mathrm{d} A(\mathrm{~d} a)$ and an absolute temperature distribution $\theta$. The gradient operator on $\mathcal{R}_{o}(\mathcal{R})$ is denoted as $\operatorname{Grad}[\bullet](\operatorname{grad}[\bullet])$ and the divergence as $\operatorname{Div}[\bullet](\operatorname{div} \bullet)$. The deformation gradient $\boldsymbol{F}=\operatorname{Grad}[\boldsymbol{x}]$, with $\boldsymbol{J}=\operatorname{det} \boldsymbol{F}$, maps the first Piola-Kirchhoff stress $\boldsymbol{P}$ to the Cauchy stress $\boldsymbol{T}=J^{-1} \boldsymbol{P} \boldsymbol{F}^{\mathrm{T}}$. The induced temperature gradient and heat flux vectors on the reference (current) configuration are denoted, respectively, as $\boldsymbol{g}_{o}=\operatorname{Grad}[\theta]\left(\boldsymbol{g}=\operatorname{grad}[\theta]=\boldsymbol{F}^{-\mathrm{T}} \boldsymbol{g}_{o}\right)$ and $\boldsymbol{q}_{o}$ $\left(\boldsymbol{q}=J^{-1} \boldsymbol{F} \boldsymbol{q}_{o}\right)$. Corresponding to these are the Piola (Cauchy) traction vector $\boldsymbol{p}=\boldsymbol{P} \boldsymbol{N}(\boldsymbol{t}=\boldsymbol{T} \boldsymbol{n})$, with pressure $p_{N}=-\boldsymbol{p} \cdot \boldsymbol{n}\left(t_{N}=-\boldsymbol{t} \cdot \boldsymbol{n}\right)$ and normal heat flux $h_{o}=-\boldsymbol{q}_{o} \cdot \boldsymbol{N}(h=-\boldsymbol{q} \cdot \boldsymbol{n})$.

For the specific class of thermomechanical problems considered, all rate and supply terms are omitted from the linear momentum and energy balance equations:

$$
\operatorname{Div}[\boldsymbol{P}]=0, \quad-\operatorname{Div}\left[\boldsymbol{q}_{o}\right]=0 .
$$

The constitutive model for the heat flux employs Fourier's law on the deformed configuration such that $\boldsymbol{q}_{o}=-J k \boldsymbol{C}^{-1} \boldsymbol{g}_{o}$ with $k$ as the conductivity from (5.1) and $\boldsymbol{C}=\boldsymbol{F}^{\mathrm{T}} \boldsymbol{F}$. Finally, the Helmholtz energy delivers the stress and is modeled according to the modified entropic theory of elasticity $[50,51]$. Note that thermomechanical coupling persists in the present quasistatic setting owing to the temperature dependence of the stress and the deformation dependence of $\boldsymbol{q}_{o}$. While the presented homogenization approach excludes rate affects such as in viscoelasticity, a thermoplastic material model may also be employed without any modifications to the overall framework.

\section{APPENDIX B: ON A FORMULATION OF MORTAR-BASED THERMAL CONTACT}

The classical steady-state linearized thermal conduction problem may be stated as the minimization of a potential. This potential may be supplemented by a thermal contact potential

$$
\mathcal{C}_{\theta}\left[\vartheta_{c}, \gamma_{\theta}, h_{o}\right]=\int_{\Gamma_{o}^{c}} \mathcal{H}_{o} \mathrm{~d} A+\int_{\Gamma_{o}^{c}} h_{o}\left(\vartheta_{c}-\gamma_{\theta}\right) \mathrm{d} A,
$$

which is motivated by the classical three-field mixed variational formulation of [52] for normal contact. For simplicity, assuming a frozen mechanical configuration, the interface dissipation potential $\mathcal{H}_{o}=\mathcal{H}_{o}\left(\gamma_{\theta}\right)$ is postulated. Here, $\mathcal{H}_{o}$ must be evaluated via $\gamma_{\theta}$ for variational consistency. Upon enforcing the stationarity of the total potential, one obtains the contribution (4.1) to the energy balance, with $h_{o}$ of yet undetermined value. Additionally, one obtains the equalities

$$
\int_{\Gamma_{o}^{c}} \delta h_{o}\left(\gamma_{\theta}-\vartheta_{c}\right) \mathrm{d} A=0, \quad \int_{\Gamma_{o}^{c}} \delta \gamma_{\theta}\left(h_{o}-\partial \mathcal{H}_{o} / \partial \gamma_{\theta}\right) \mathrm{d} A=0 .
$$

If $\left\{\vartheta_{c}, \gamma_{\theta}, h_{o}\right\}$ have infinite degrees of freedom on $\Gamma_{o}^{c}, \gamma_{\theta}=\vartheta_{c}$ is implied by the first equality, and the classical continuum formulation (4.2) is recovered from the second. However, $\vartheta_{c}$ varies according to the discretizations of the master and slave surfaces, whereas $\left\{\gamma_{\theta}, h_{o}\right\}$ inherit the discretization of the slave according to (4.4), thereby inducing (4.5). This formulation addresses the discretization 
associated with the modeling of thermal contact independent of any constraint idea. In particular, it is valid also for the constitutive model of $\mathcal{H}_{o}$. In that case, the pressure in $\mathcal{H}_{o}$ is kept fixed during the variation, similar to the treatment of the frictional slip contribution in the variational setting of [45], but contributes to subsequent consistent linearization. Overall, this formulation offers a convenient framework for the derivation of the mortar-based approach presented in Section 4.2. See [32] for a similar derivation in the case of frictionless contact.

When $\mathcal{H}_{o}=-\epsilon_{\theta} \gamma_{\theta}^{2} / 2$, the penalty method acts as the underlying constraint enforcement methodology with $\epsilon_{\theta}$ as the penalty parameter. To extend the formulation to the numerically superior augmented Lagrangian setting in this constraint case, it is sufficient to augment $\mathcal{C}_{\theta}$ by classical terms:

$$
\mathcal{C}_{\theta}^{\lambda}\left[\lambda_{\theta}, \vartheta_{c}, \gamma_{\theta}, h_{o}\right]=\mathcal{C}_{\theta}\left[\vartheta_{c}, \gamma_{\theta}, h_{o}\right]+\int_{\Gamma_{o}^{c}} \lambda_{\theta} \gamma_{\theta} \mathrm{d} A+\int_{\Gamma_{o} \backslash \Gamma_{o}^{c}} \frac{\lambda_{\theta}^{2}}{2 \epsilon_{\theta}} \mathrm{d} A
$$

When taken as the starting point, the variations of $\mathcal{C}_{\theta}^{\lambda}$ yield (4.9) and (4.10) in addition to (4.5) 1 .

\section{REFERENCES}

1. Persson BNJ. Sliding Friction: Physical Principles and Applications, 2nd edition. Springer: Berlin Heidelberg New York, 2000.

2. Szeri AZ. Fluid Film Lubrication, 2nd edition. Cambridge University Press: Cambridge, 2011.

3. Madhusudana CV. Thermal Contact Conductance. Springer: New York, 1996.

4. Available from: http://www.electronics-cooling.com/ accessed on 1 September 2012.

5. Prasher R. Thermal interface materials: historical perspective, status and future directions. Proceedings of the IEEE 2006; 94(8): 1571-1586.

6. Baek SS, Fearing RS. Reducing contact resistance using compliant nickel nanowire arrays. IEEE Transactions on Components and Packaging Technologies 2008; 31:859-868.

7. Barisik M, Beskok A. Boundary treatment effects on molecular dynamics simulations of interface thermal resistance. Journal of Computational Physics 2012; 231:7881-7892.

8. Xiang H, Jiang P-X, Liu Q-X. Non-equilibrium molecular dynamics study of nanoscale thermal contact resistance. Molecular Simulation 2010; 34:679-687.

9. Persson BNJ, Lorenz B, Volokitin AI. Heat transfer between elastic solids with randomly rough surfaces. European Physical Journal E: Soft Matter 2010; 31:3-24.

10. Prasher RS, Phelan PE. Microscopic and macroscopic thermal contact resistances of pressed mechanical contacts. Journal of Applied Physics 2006; 100:063538.

11. Bahrami M, Culham JR, Yovanovich MM, Schneider GE. Review of thermal joint resistance models for nonconforming rough surfaces. Applied Mechanics Reviews 2006; 59:1-12.

12. Bahrami M, Yovanovich MM, Marotta EE. Thermal joint resistance of polymer-metal rough interfaces. Journal of Electronic Packaging 2006; 128:23-29.

13. Kim I-C, Marotta EE, Fletcher LS. Thermal joint conductance of low-density polyethylene and polyester polymeric films: experimental. Journal of Thermophysics and Heat Transfer 2006; 20:398-407.

14. Paggi M, Barber JR. Contact conductance of rough surfaces composed of modified RMD patches. International Journal of Heat and Mass Transfer 2011; 54:4664-4672.

15. Savija I, Culham JR, Yovanovich MM. Effective thermophysical properties of thermal interface materials: part I-definitions and models. Proceedings of InterPACK2003: International Electronic Packaging Technical Conference and Exhibition, Maui, Hawaii, USA, July 6-11, 2003; 35088.

16. Savija I, Culham JR, Yovanovich MM. Effective thermophysical properties of thermal interface materials: part II-experiments and data. Proceedings of InterPACK2003: International Electronic Packaging Technical Conference and Exhibition, Maui, Hawaii, USA, July 6-11, 2003; 35264.

17. Temizer İ. Thermomechanical contact homogenization with random rough surfaces and microscopic contact resistance. Tribology International 2011; 44:114-124.

18. Sadowski P, Stupkiewicz S. A model of thermal contact conductance at high real contact area fractions. Wear 2010; 268:77-85.

19. Salti B, Laraqi N. 3-D numerical modeling of heat transfer between two sliding bodies: temperature and thermal contact resistance. International Journal of Heat and Mass Transfer 1999; 42:2363-2374.

20. Thompson MK. A multi-scale iterative approach for finite element modeling of thermal contact resistance. Ph.D. Thesis, Massachusetts Institute of Technology, Boston, Massachusetts (USA), 2007.

21. Temizer İ, Wriggers P. Thermal contact conductance characterization via computational contact homogenization: a finite deformation theory framework. International Journal for Numerical Methods in Engineering 2010; 83:27-58.

22. Barber JR. Indentation of a semi-infinite elastic solid by a hot sphere. International Journal of Engineering Science 1973; 15:813-819. 
23. Budt M, Temizer İ, Wriggers P. A computational homogenization framework for soft elastohydrodynamic lubrication. Computational Mechanics 2012; 49:749-767.

24. Temizer İ. On the asymptotic expansion treatment of two-scale finite thermoelasticity. International Journal of Engineering Science 2012; 53:74-84.

25. Hughes TJR, Cottrell JA, Bazilevs Y. Isogeometric analysis: CAD, finite elements, NURBS, exact geometry and mesh refinement. Computer Methods in Applied Mechanics and Engineering 2005; 194:4135-4195.

26. Hüeber S, Mair M, Wohlmuth BI. A priori error estimates and an inexact primal-dual active set strategy for linear and quadratic finite elements applied to multibody contact problems. Applied Numerical Mathematics 2005; 54:555-576.

27. Puso MA, Laursen TA, Solberg J. A segment-to-segment mortar contact method for quadratic elements and large deformations. Computer Methods in Applied Mechanics and Engineering 2008; 197:555-566.

28. De Lorenzis L, Wriggers P, Zavarise G. A mortar formulation for 3D large deformation contact using NURBS-based isogeometric analysis and the augmented Lagrangian method. Computational Mechanics 2012; 49:1-20.

29. Hesch C, Betsch P. Isogeometric analysis and domain decomposition methods. Computer Methods in Applied Mechanics and Engineering 2012; 213-216:104-112.

30. Kim J-Y, Youn S-K. Isogeometric contact analysis using mortar method. International Journal for Numerical Methods in Engineering 2012; 89:1559-1581.

31. Lu J. Isogeometric contact analysis: geometric basis and formulation of frictionless contact. Computer Methods in Applied Mechanics and Engineering 2011; 200:726-741.

32. Temizer İ. A mixed formulation of mortar-based frictionless contact. Computer Methods in Applied Mechanics and Engineering 2012; 223-224:173-185.

33. Temizer İ, Wriggers P, Hughes TJR. Three-dimensional mortar-based frictional contact treatment in isogeometric analysis with NURBS. Computer Methods in Applied Mechanics and Engineering 2012; 209-212:115-128.

34. Wohlmuth B. Variationally consistent discretization schemes and numerical algorithms for contact problems. Acta Numerica 2011; 20:569-734.

35. Puso MA, Laursen TA. A mortar segment-to-segment contact method for large deformations. Computer Methods in Applied Mechanics and Engineering 2004; 193:601-629.

36. Puso MA, Laursen TA. A mortar segment-to-segment frictional contact method for large deformations. Computer Methods in Applied Mechanics and Engineering 2004; 193:4891-4913.

37. Hesch C, Betsch P. Energy-momentum consistent algorithms for dynamic thermomechanical problems—application to mortar domain decomposition problems. International Journal for Numerical Methods in Engineering 2011; 86:1277-1302.

38. Hüeber S, Wohlmuth BI. Thermo-mechanical contact problems on non-matching meshes. Computer Methods in Applied Mechanics and Engineering 2009; 198:1338-1350.

39. Laursen TA, Puso MA, Sanders J. Mortar contact formulations for deformable-deformable contact: past contributions and new extensions for enriched and embedded interface formulations. Computer Methods in Applied Mechanics and Engineering 2012; 205-208:3-15.

40. Laursen TA. Computational Contact and Impact Mechanics, 1st edition. Springer: Berlin Heidelberg New York, 2003. (corr. 2nd printing).

41. Wriggers P. Computational Contact Mechanics, 2nd edition. Springer: Berlin Heidelberg New York, 2006.

42. Agelet de Saracibar CA. Numerical analysis of coupled thermomechanical frictional contact problems. Computational model and applications. Archives of Computational Methods in Engineering 1999; 5:243-301.

43. Strömberg N, Johansson L, Klarbring A. Derivation and analysis of a generalized standard model for contact, friction and wear. International Journal for Solids and Structures 1996; 33:1817-1836.

44. Zavarise G, Wriggers P, Stein E, Schrefler BA. Real contact mechanisms and finite element formulation-a coupled thermomechanical approach. International Journal for Numerical Methods in Engineering 1992; 35:767-785.

45. Alart P, Curnier A. A mixed formulation for frictional contact problems prone to Newton like solution methods. Computer Methods in Applied Mechanics and Engineering 1991; 92:353-375.

46. Gitterle M, Popp A, Gee MW, Wall WA. Finite deformation frictional mortar contact using a semi-smooth Newton method with consistent linearization. International Journal for Numerical Methods in Engineering 2010; 84:543-571.

47. Comninou M, Dundurs J, Barber JR. Planar Hertz contact with heat conduction. Journal of Applied Mechanics 1981; 48:549-554.

48. Comninou M, Barber JR. The thermoelastic Hertz problem with pressure dependent contact resistance. International Journal of Mechanical Sciences 1984; 26:549-554.

49. Konyukhov A, Schweizerhof K. Incorporation of contact for high-order finite elements in covariant form. Computer Methods in Applied Mechanics and Engineering 2009; 198:1213-1223.

50. Chadwick P. Thermo-mechanics of rubberlike materials. Philosophical Transactions of the Royal Society of London, Series A 1973; 276:371-403.

51. Chadwick P, Creasy CFM. Modified entropic elasticity of rubberlike materials. Journal of the Mechanics and Physics of Solids 1984; 32(5):337-357.

52. Papadopoulos P, Taylor RL. A mixed formulation for the finite element solution of contact problems. Computer Methods in Applied Mechanics and Engineering 1992; 94:373-389. 